Energy Aware Basestation Placement in

Solar Powered Sensor Networks 


\title{
ENERGY AWARE BASESTATION PLACEMENT IN SOLAR POWERED SENSOR NETWORKS
}

\author{
BY \\ SAYED-ALI SHARIATMADARI, B.Sc.
}

\begin{abstract}
A THESIS
SUBMITTED TO THE DEPARTMENT OF ELECTRICAL \& COMPUTER

ENGINEERING

AND THE SCHOOL OF GRADUATE STUDIES

OF MCMASTER UNIVERSITY

IN PARTIAL FULFILMENT OF THE REQUIREMENTS

FOR THE DEGREE OF

MASTER OF APPLIED SCIENCE
\end{abstract}

(C) Copyright by Sayed-Ali Shariatmadari, August 2010 All Rights Reserved 
Master of Applied Science (2010)

(Electrical \& Computer Engineering)
McMaster University

Hamilton, Ontario, Canada

TITLE:

Energy Aware Basestation Placement in Solar Powered Sensor Networks

AUTHOR:

Sayed-Ali Shariatmadari

B.Sc., (Electrical Engineering)

Isfahan University of Technology, Isfahan, Iran

SUPERVISOR: $\quad$ Dr. Terence D. Todd

NUMBER OF PAGES: $\quad x, 75$ 
To my fellow Iranians who were murdered or arrested in the protests following the 2009 Iranian presidential election. 


\section{Abstract}

Sensor nodes are often used in outdoor locations where they can be operated using solar power. When such a network is deployed, there are usually restrictions in the way that the nodes can be positioned, and this results in a node-dependent attenuation of the usable solar energy. This effect must be taken into account when placing the basestations used to communicate with the sensor nodes. In this thesis we consider the minimum-cost placement of data collecting basestation nodes so that outage-free operation of the sensor nodes is obtained. This is done by minimizing the number of basestations required when taking into account the energy costs of sensor node traffic relaying. An optimization is first formulated which gives a lower bound on the number of basestations that are required. Because of the complexity of the problem, two algorithms are proposed which can be used to do placements for practical problem sizes. These algorithms use the result from an iterated local search as a starting point, and then use an energy aware local optimization to obtain feasible basestation placements. Results are presented which show that the algorithms perform well for a variety of network scenarios. 


\section{Acknowledgements}

I would like to express my sincere and honest thanks to my supervisor, Prof. Terence D. Todd for his guidance and support throughout my graduate studies at McMaster University. Indeed, his careful supervision and consistent help and support helped me find my interests and made it possible for me to finish this work.

I am very grateful to Prof. Ted Szymanski and Prof. Dongmei Zhao for reviewing my thesis and for their valuable suggestions and comments. Also, I would like to thank all the members of the Wireless Communication Laboratory for their helpful discussions and suggestions.

Finally, I would like to give my special thanks to my wife and my parents, for their encouragement and support. 


\section{Contents}

Abstract iv

Acknowledgements $\quad$ v

1 Introduction 3

1.1 Power Management in Wireless Sensor Networks . . . . . . . . 3

1.2 Basestation Placement in Wireless Sensor Networks . . . . . . 5

1.3 Solar Powered Networks . . . . . . . . . . . . . . . . . 5

1.4 Optimization Theory . . . . . . . . . . . . . 7

1.4.1 Convex Optimization Problem ............ . 8

1.4.2 Linear Programming . . . . . . . . . . . . . 8

1.4.3 Mixed-Integer Programming . . . . . . . . . . . . 9

1.4.4 Feasibility problems ................ 9

1.5 Contribution . . . . . . . . . . . . . . 10

2 Power Management in Wireless Sensor Networks 12

2.1 Hardware Design and Deployment . . . . . . . . . . . . . . . 12

2.2 Simulation Framework . . . . . . . . . . . . . . . 15

2.3 Communication . . . . . . . . . . . . . 16 
2.4 Basestation Placement . . . . . . . . . . . . . . . . 18

3 Solar Powered Networks $\quad 22$

3.1 Introduction ..................... 22

3.2 Solar Radiation Data . . . . . . . . . . . . . . 23

3.3 Hardware Design of Solar Sensors . . . . . . . . . . . . 23

3.4 Energy Aware Design Challenges . . . . . . . . . . . . . . 24

3.5 Solar Based Routing Algorithms . . . . . . . . . . . . . 27

3.6 IEEE 802.11 Mesh Networks . . . . . . . . . . . . . . . . 28

4 Problem̂n Definition 33

4.1 Introduction ................... 33

4.2 Problem Formulation and Lower Bound . . . . . . . . . . . . 35

4.3 Energy Aware Basestation Placement . . . . . . . . . . . . 43

4.3.1 Initial Placement of Basestations . . . . . . . . . 45

4.3.2 Complexity of EA Algorithms . . . . . . . . . . . 46

5 Performance Results $\quad 48$

5.1 Introduction $\ldots \ldots \ldots \ldots \ldots$

5.2 Design Example . . . . . . . . . . . . . . . . . . . 48

5.2.1 The Size of Battery and Solar Panel . . . . . . . . . . . . 49

5.2.2 Heterogeneous Solar Factor . . . . . . . . . . . . . 51

5.2.3 Performance of EA Algorithms . . . . . . . . . . . 51

5.3 Larger Networks . . . . . . . . . . . . . . . 55

5.3.1 Geographical Distribution of Sensor Nodes . . . . . . . 56

5.3 .2 Solving Time . . . . . . . . . . . . 57 
5.3 .3 Results . . . . . . . . . . . . . . . . . . . . . 59

6 Conclusion and Future Works 65 


\section{List of Figures}

1.1 A Wireless Sensor Network for a Combat Filed Monitoring Application. ......................... 4

2.1 Mica Hardware Platform: The Mica Sensor Node (left) with the Mica Weather Board Developed for Environmental Monitoring Applications .......................... 13

2.2 System Architecture for Habitat Monitoring . . . . . . . . . . . . 14

2.3 Multi-Gateway Clustered Sensor Network . . . . . . . . . . 18

2.4 Reference Architecture for a Two-Tiered Wireless Sensor Network. 19

2.5 The disk is divided into 16 subareas for the 3-node example. . . 20

3.1 Interactions Between Various EEHF Algorithms . . . . . . . 25

3.2 Best-effort mesh AP power saving with movable boundary. . . . 29

3.3 Forced mesh AP power saving (FPS) with $50 \%$ offered capacity. 30

3.4 Solar Powered WLAN Mesh Node . . . . . . . . . . . . . . . . . 31

4.1 Basestations and Solar Powered Sensor Nodes. Region I has a lower solar factor than Region II. The deployed basestation density is lower in Region II. . . . . . . . . . . . . . . . . . . . 34

5.1 Design Example. . . . . . . . . . . . . . . . . . 49 
5.2 Comparing Node Placement in a Homogeneous Solar Energy network vs a Heterogeneous one . . . . . . . . . . . . . 52

5.3 Different Network Topologies . . . . . . . . . . . . . . . 57

5.4 Problem Solve Time of Different Topologies and Flow Volume . 58

5.5 Results for a Uniform Distribution when Flow Volume is 1 unit 60

5.6 Results for a Uniform Distribution when Flow Volume is 0.5 unit 61

5.7 Results for a Normal Distribution when Flow Volume is 1 unit . 62

5.8 Results for a Normal Distribution when Flow Volume is 0.5 unit 63 


\section{List of Tables}

4.1 Notations . . . . . . . . . . . . . . . . 36

5.1 Modeling Parameters . . . . . . . . . . . . . . . . . . 50

5.2 The Number of Placed Basestation vs The Volume of Traffic that is Generated by Nodes. . . . . . . . . . . . . . . . . . 50

5.3 The Number of Placed Basestation vs The Volume of Traffic that is Generated by Nodes. . . . . . . . . . . . . . . . . . . . . 51

5.4 Number of Basestations: Link Capacity vs. Solar Factor. Results of Lower Bound and Local Search Algorithms. . . . . . . . . . 53

5.5 Number of Basestations: Link Capacity vs. Solar Factor. Results of Minimum Load and Minimum Battery Usage Algorithms. . .

5.6 Number of Basestations: Battery Capacity vs. Solar Factor. Results of Lower Bound and Local Search Algorithms. . . . . . . . 55

5.7 Number of Basestations: Battery Capacity vs. Solar Factor. Results of Minimum Load and Minimum Battery Usage Algorithms. 56

5.8 The Coefficient of Variation of Solving Time . . . . . . . . . . . 59

5.9 Degree of competence of two EA algorithms . . . . . . . 60 


\section{List of Algorithms}

4.1 Energy Aware (EA) Algorithm, Minimum Load . . . . . . . . 44

4.2 Iterative Local Search (to initiate EA Algorithm) . . . . . . . . 46 


\section{Chapter 1}

\section{Introduction}

Increasing interest in Wireless Sensor Networks has been seen in the past years. Their relatively cheap price permits the deployment of hundreds of sensor nodes in a single network. Wireless Sensors Networks can be used for many purposes such as military applications, environmental and industrial monitoring, vehicle detection and agriculture [1, 2, 3]. A sample wireless sensor network that monitors a combat field is shown in Figure 1.1. In this network a basestation is deployed near the sensors to connect the network to remote command centers [4].

\subsection{Power Management in Wireless Sensor Networks}

Wireless sensor nodes are micro-electronic devices and have a very limited source of power. They are commonly powered using batteries, but for applications where the system is expected to operate for a long period, energy 
M.A.Sc. Thesis - Sayed-Ali Shariatmadari McMaster - Electrical Engineering

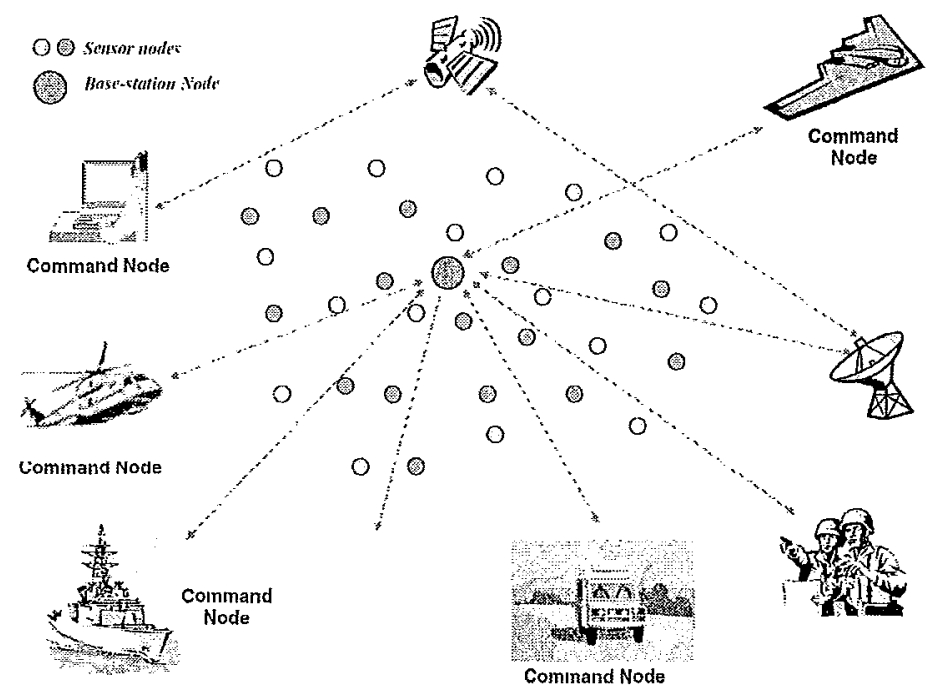

Figure 1.1: A Wireless Sensor Network for a Combat Filed Monitoring Application.

becomes a bottleneck. In sensor networks, normally each sensor can relay traffic to other sensors using multi-hop routing algorithms until this data reaches its destination.

A lot of effort has been made to efficiently use battery energy. In multihop sensor networks where sensors generate and route the data, power conservation and power management plays an important role. Because a group of dysfunctional nodes changes the routing algorithm and may require network reorganization and retransmission of data, the design of power-aware protocols and algorithms for sensor networks is vital [5]. 
M.A.Sc. Thesis - Sayed-Ali Shariatmadari McMaster - Electrical Engineering

\subsection{Basestation Placement in Wireless Sensor Net- works}

Basestation location has a significant impact on network lifetime performance for a sensor network. The main job of a sensor node is to sense events, process gathered data, and transmit the data to an appropriate gateway. Therefore, power consumption consists of three parts: sensing, data processing and communication. Communication consumes the maximum energy among these three sources [5].

Transmission energy consumption between two nodes depends not only on the data bit rate but also physical distance between them [6]. This means that the location of nodes and basestations in a sensor network affects network lifetime and performance and for this reason, optimizing network topology is very important.

\subsection{Solar Powered Networks}

Solar power is increasingly used to operate sensors and other mesh nodes in a variety of different outdoor applications [7]. In a typical scenario, sensor nodes communicate to the Internet through a deployment of fixed basestation (BS) nodes, which are usually equipped with continuous power connections. In this type of arrangement, some sensor nodes may be able to communicate directly with a basestation, whereas others may have to use multi-sensor traffic 
M.A.Sc. Thesis - Sayed-Ali Shariatmadari McMaster - Electrical Engineering

relaying in order to do so. Since the solar panel and node battery configuration is typically done in a statistical fashion, care must be taken so that once deployed, there is sufficient solar insolation so that the nodes can operate continuously without outage [8].

A vital difference between this kind of system and battery powered ones is that the amount of energy that can be harvested from the environment can be unlimited. A harvesting network can be built by using energy-harvesting nodes [9]. In addition, in a battery powered sensor network, the design goal is to maximize the lifetime or to minimize the power consumption of the nodes. However, in energy-harvesting networks, the goal is to design the network and allocate resources (battery and panel sizes) in a way that reduces costs while guaranteeing outage free operation of the nodes.

In traditional photo-voltaic systems, solar panels are positioned so that they can collect the maximum possible solar energy. This is done by orienting the panel directly south (in the northern hemisphere) and sloped to an angle slightly less than the node's geographic latitude, so that solar absorption is maximized during winter months. Unfortunately this requirement places severe restrictions on the location and the flexibility with which the nodes may be placed. This type of restriction is unreasonable in many sensor applications, since the positioning and orientation of the nodes is usually constrained by other factors [7]. A simple example is the case where a sensor node must be installed on the side of a building, and due to its non-optimum orientation, may suffer orientation and shadowing losses from the direct components of solar insolation, compared with an optimally-positioned node. In this case the 
M.A.Sc. Thesis - Sayed-Ali Shariatmadari McMaster - Electrical Engineering

shadowing components may also be time varying, and dependent upon the orientation of the building wall. These effects lead to a wide variation in the efficiencies with which the sensor nodes can collect solar energy.

The above problem is exacerbated by the fact that to exploit economies of scale, the sensor nodes are often equipped with a standard battery and solar panel configuration, so that the costs of the nodes are minimized. This places an onus on the deployment of the basestations so that their number and location is sufficient to ensure outage-free operation of the sensor nodes regardless of their individual placements.

\subsection{Optimization Theory}

In this thesis, optimization theory is used to do basestation placement. Optimization theory has included significant advances in the last two decades. Solvers using methods such as interior-point method, conic optimization and branch-and-bound are getting faster and better. The standard form of an optimization problem can be written as [10],

$$
\begin{array}{ll}
\operatorname{minimize} & f_{0}(x) \\
\text { subject to } & f_{i}(x) \leq 0, \quad i=1 \ldots m \\
& h_{i}(x)=0, \quad i=1 \ldots p
\end{array}
$$

$x$ is the optimization variable and $f_{0}$ is the objective function. The inequalities $f_{i}(x) \leq 0$ are called inequality constraints and the equations $h_{i}(x)=0$ are called the equality constraints. The problem (1.1) is said to be feasible if there 
M.A.Sc. Thesis - Sayed-Ali Shariatmadari McMaster - Electrical Engineering

exists at least one feasible point, and infeasible otherwise.

\subsubsection{Convex Optimization Problem}

In convex optimization, objective function and constraints are convex. A convex optimization problem is of the form

$$
\begin{array}{ll}
\operatorname{minimize} & f_{0}(x) \\
\text { subject to } & f_{i}(x) \leq 0, \quad i=1 \ldots m \\
& a_{i}^{T} x=b_{i}, \quad i=1 \ldots p
\end{array}
$$

where $f_{0}, \ldots, f_{m}$ are convex functions. The importance of convex optimization is that any local optimum is also globally optimal. Convex optimization is widely accepted as a method for designing of communication systems [11].

\subsubsection{Linear Programming}

In an optimization problem, when the objective and constraint functions are all affine, the problem is called a linear program (LP). A general linear program has the form

$$
\begin{array}{cl}
\operatorname{minimize} & c^{T} x \\
\text { subject to } & G x \leq h \\
& A x=b
\end{array}
$$

where $G \in R^{m \times n}$ and $A \in R^{p \times n}$. Linear programs are convex optimization problems. 
M.A.Sc. Thesis - Sayed-Ali Shariatmadari McMaster - Electrical Engineering

\subsubsection{Mixed-Integer Programming}

A mixed-integer optimization problem is an optimization problem in which some of the optimization variables are integer-valued, while the rest are realvalued. Mixed-integer optimization problems are usually difficult to solve. In particular, they cannot be solved within polynomial time. In fact, the complexity of integer and mixed-integer optimization problems is NP-hard [12]. That is, finding the optimal solution may require examining all the feasible points using exhaustive search. There are several commercial packages that can solve these kinds of problems (e.g., CPLEX [13] or GUROBI [14]), which is commonly done using the branch and bound method.

\subsubsection{Feasibility problems}

If the objective function is zero, the optimal value is either zero (if the feasible set is nonempty) or inf (if the feasible set is empty). This type of problem is called feasibility problem. It also can be written as

$$
\begin{aligned}
\text { find } & x \\
\text { subject to } & f_{i}(x)<0, \quad i=1 \ldots m \\
& h_{i}(x)<0, \quad i=1 \ldots p
\end{aligned}
$$

Thus the problem is if the constraints are consistent, find a point that satisfies them. 
M.A.Sc. Thesis - Sayed-Ali Shariatmadari McMaster - Electrical Engineering

\subsection{Contribution}

In this thesis the placement of basestations from a renewable energy point of view is considered. To accommodate the solar power assumption, the network design must be done using historical solar insolation sample functions for the region where the network is to be deployed. In our results we use data for Toronto, Canada, since it is typical of a temperate continental climate. The objective is to find the best basestation deployment so that when the system is subject to the design bandwidth usage profile and solar insolation sample functions, the network survives outage-free. This is a unique aspect of the problem which must take into account the energy input/output recursion at each sensor node.

The basestation nodes are placed so that outage-free operation of the sensors is ensured. This is accomplished by minimizing the number of basestations that are needed when taking into account the energy needed for the sensor nodes to perform traffic relaying. The problem is first formulated as a discrete optimization, which gives a lower bound on the number of basestations that are needed. Due to the complexity of the problem, an algorithm is proposed which can be used to do placements for practical problem sizes. The algorithm uses an iterated local search to find a starting point, and then uses an energy aware local optimization to obtain feasible basestation placements. Results are presented which show that the proposed algorithm performs well for a variety of network scenarios.

The rest of the thesis is organized as follows. In Chapter 2 we review latest researches on battery powered hardware design and power management 
M.A.Sc. Thesis - Sayed-Ali Shariatmadari McMaster - Electrical Engineering

methods in wireless sensor networks. In Chapter 3 we briefly review solar powered sensor and mesh networks. In Chapter 4 we formulate our problem and then discuss its complexity and then propose algorithms with reasonable running time. Chapter 5 has the performance results of proposed algorithms and comparison with optimal solutions. Chapter 6 summarizes our work and proposes some future research topics. Preliminary work of this thesis is published as a conference paper in IEEE Wireless Communications and Networking Conference, Sydney, Australia, April 2010 [15]. 


\section{Chapter 2}

\section{Power Management in Wireless}

\section{Sensor Networks}

Wireless sensor networks are an effective way of monitoring environments. However, one of the major challenges in designing these networks is the limited energy available to sensor nodes. For this reason, a large fraction of studies in wireless sensor networks are focused on the problem of energy efficiency. They use the fact that data transmission and radio communication by orders of magnitude are more expensive than computation. This trade-off has a large impact on the design of hardware, algorithms and network platforms in sensor networks.

\subsection{Hardware Design and Deployment}

New developments in wireless communications have resulted in smaller, lowpower and low-cost sensors. The Mica platform, designed by researchers at 
M.A.Sc. Thesis - Sayed-Ali Shariatmadari McMaster - Electrical Engineering

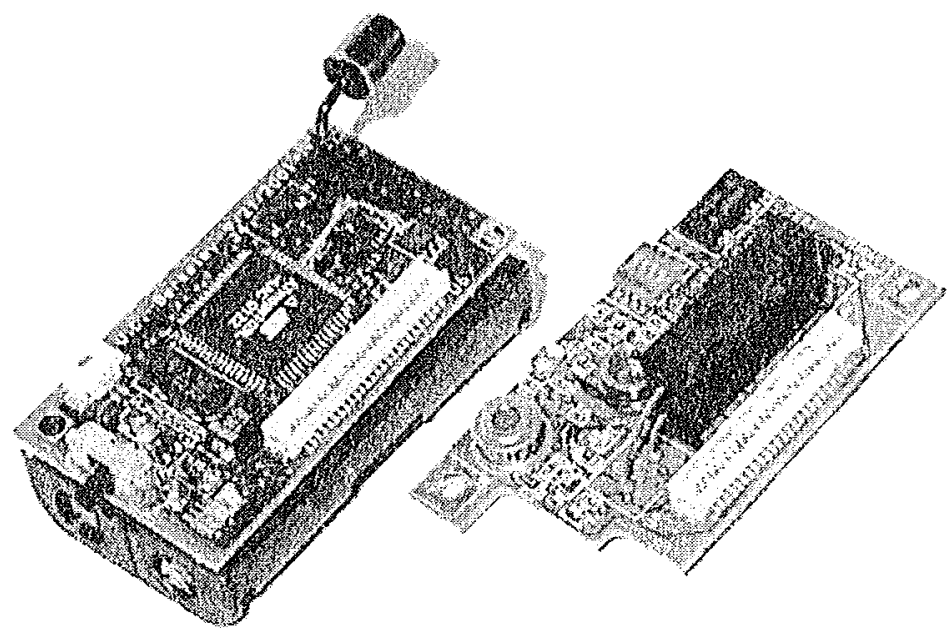

Figure 2.1: Mica Hardware Platform: The Mica Sensor Node (left) with the Mica Weather Board Developed for Environmental Monitoring Applications

the University of California, Berkeley, introduced a new approach in wirelesssystem design of low cost embedded devices. It is suitable for a self-configuring multihop (mesh) network platform for remote monitoring [16]. The Mica platform, pictured in Figure 2.1, uses an operating system called TinyOS that is specifically designed for deeply embedded systems with minimal hardware.

An architecture of a habitat monitoring network using Mica devices, commonly known as "motes", is shown in Figure 2.2. System design requirements are reviewed in Reference [17]. This architecture is implemented as a 32 node network using a tiered architecture. The first level are sensor nodes that gather data. Then this data is transmitted to the network gateway. The gateway is responsible for transmitting data through a local network to the remote basestation. The basestation is connected to database servers across the internet. This data is logged in those servers and is displayed through a user interface. Mobile devices can also interact with the network. 
M.A.Sc. Thesis - Sayed-Ali Shariatmadari McMaster - Electrical Engineering

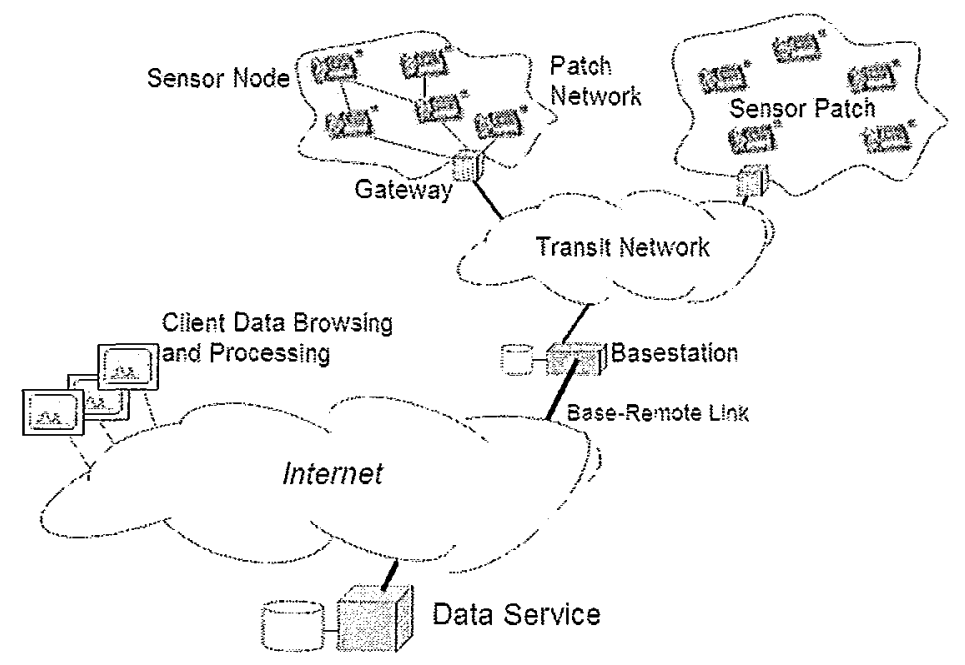

Figure 2.2: System Architecture for Habitat Monitoring

An intelligent wireless sensor node using a programmable system on a chip is introduced in Reference [18]. This work uses a low-power programmable system on chip to reduce energy consumption of sensors. These sensors are used in a heart disease monitoring system. Having the capability of processing heavy computation and complex functions rapidly helps to process the gathered data at the sensor itself instead of sending it to a base station. This approach reduces the amount of transmitted data and saves energy at nodes and increases the node lifetime.

Reference [19] is focused on the storage needs of sensor networks. Local storage is used in many sensor networks for either saving gathered data or for memory. This works uses the recent gains in energy efficiency of newgeneration NAND flash storage. It is argued that current solutions for data storage in sensor networks which only provide a simple file system are not competent enough. Instead a new object storage abstraction named Capsule 
M.A.Sc. Thesis - Sayed-Ali Shariatmadari McMaster - Electrical Engineering

is introduced. Capsule provides stream, file, array, queue and index storage objects. It also supports checkpoints and rollback of objects. It is argued that Capsule provides better energy efficiency than current storage solutions.

One way to deploy a large number of sensor nodes is to deploy them in clusters. But in this method, the location of a particular sensor cannot be guaranteed. This increases the number of nodes that are needed to completely cover the monitored area. In Reference [20] a heuristic is discussed that selects mutually exclusive sets of sensor nodes where each set can completely cover the area. Activating only one set is enough and this helps saving node energy.

\subsection{Simulation Framework}

Sensor nodes are tiny sensing and computing devices with limited energy, computational and communicational resources. In addition, sensor networks are often deployed in outdoor environments which are uncontrolled. These networks exploit distributed algorithms for efficient data processing. Algorithms cannot be implemented without considering real world impact and debugging these algorithms is very difficult. For example, a breakpoint can invalidate the gathered data. Therefore new tools are needed to aid programmers in developing applications for sensor networks. To ease developing algorithms for sensor networks, an event-driven simulation environment for TinyOS applications, TOSSIM, was developed [21]. Also an extension to TOSSIM to address the aspect of power consumption was developed in Reference [22]. This extension, PowerTOSSIM, tries to consider low-level energy requirements of the CPU, radio, sensors and other peripherals. This is done by estimating the 
M.A.Sc. Thesis - Sayed-Ali Shariatmadari McMaster - Electrical Engineering

number of CPU cycles executed by each node. It also has a model of energy consumption base on Mica2 sensor node platform.

\subsection{Communication}

Advances in radio technology enable small and cheap nodes that minimize energy consumption of data transmission. The modulation and transmission strategy play a vital role in this when applications must satisfy given throughput and delay requirements. In Reference [23] the authors show that when total energy consumption which includes both transmission energy and circuit energy is considered, the traditional belief that multi-input-multi-output (MIMO) systems are more efficient that single-input-single-output (SISO) systems are not always correct. In short range applications with fixed modulation and data rate SISO systems may outperform a MIMO system.

A phenomenon that impacts communication performance in wireless sensor networks is radio irregularity. Variance in RF sending power and different path losses are some factors that cause these irregularities. In Reference [24] first a radio simulation model called Radio Irregularity Model (RIM), is established. This model is based on data gathered from the MICA2 platform and is used to analyze the impact of radio irregularity on MAC and routing protocols. It was shown that radio irregularity has large impact on routing protocols but a small impact on MAC protocols. To deal with radio irregularity, some solutions are proposed and evaluated.

Reference [4] considers an energy-aware approach for the management of sensor networks that maximizes the lifetime of the sensors. This approach 
M.A.Sc. Thesis - Sayed-Ali Shariatmadari McMaster - Electrical Engineering

dynamically sets routes and chooses media access to minimize energy consumption. It divides the network into clusters and then assigns a node that is less energy constrained as cluster manager. A time-based MAC protocol is also described and discussed.

Two data gathering and aggregation protocols are proposed in Reference [25]. It tries to be a near optimal spanning tree routing protocol. These protocols performed well in networks where the basestation is far away and where it is in the center of the field. PEDAP (Power Efficient Data gathering and Aggregation Protocol) shows a good improvement over other similar protocols.

Reference [26] studies a power management protocol that is distributed and on demand. It provides routing information to nodes and also shuts them down when they are not active, to help reducing up to $83 \%$ of node power consumption.

A data gathering protocol named Dozer that is designed for an environmental monitoring network is discussed in Reference [27]. An environmental monitoring network is supposed to operate continuously for several years. Minimizing communication cost helps achieving this goal. Dozer uses MAC layer, topology control, and routing all coordinated to minimize energy consumption of the sensor nodes. The network is structured as a tree and packets are transferred reliably. This application does not try to reduce the latency of data transfer. This is the price that is paid for reduction of the duty cycles of nodes. Therefore this architecture is not suitable for delay critical applications.

An approach for using a cluster based routing algorithm is proposed in Reference [28]. A network is divided into clusters and then a centralized node 


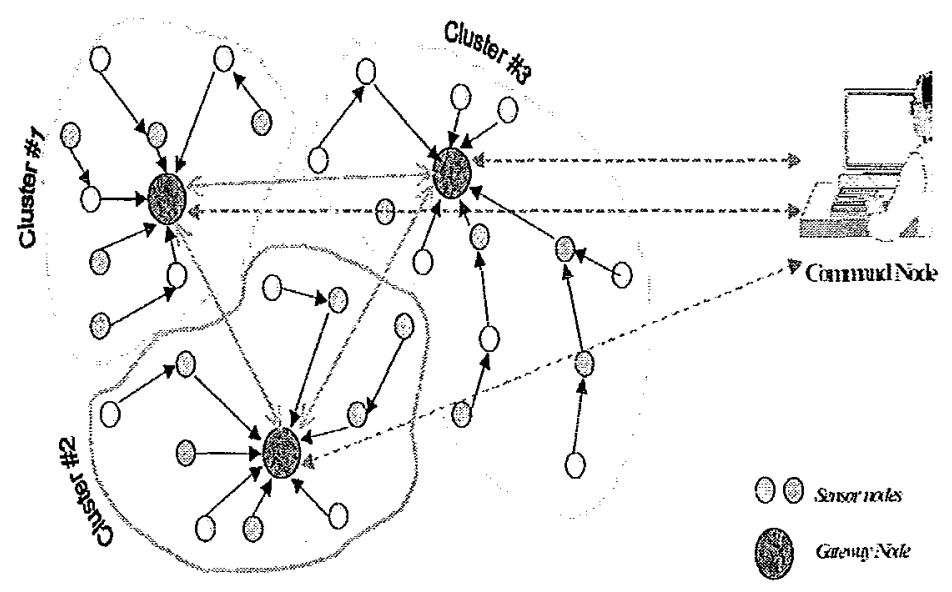

Figure 2.3: Multi-Gateway Clustered Sensor Network

is selected based on energy constraints. This allows the system to handle additional load and cover a large area. A multi-gateway clustered sensor network is shown in Figure 2.3. This node acts as a network manager, and routes for transferring data are chosen based on energy usage of the nodes and changes in the environment.

\subsection{Basestation Placement}

Base station location has a significant impact on network lifetime performance for a sensor network. This comes from the fact that data transmission and radio communication by orders of magnitude are more expensive than computation. It also has a big impact in design goals of a sensor network such as data latency and data integrity. In Reference [29] a survey is done which discusses static and dynamic strategies for node positioning. In References [30] and [31], mobile basestations and basestation repositioning is considered. 
M.A.Sc. Thesis - Sayed-Ali Shariatmadari McMaster - Electrical Engineering

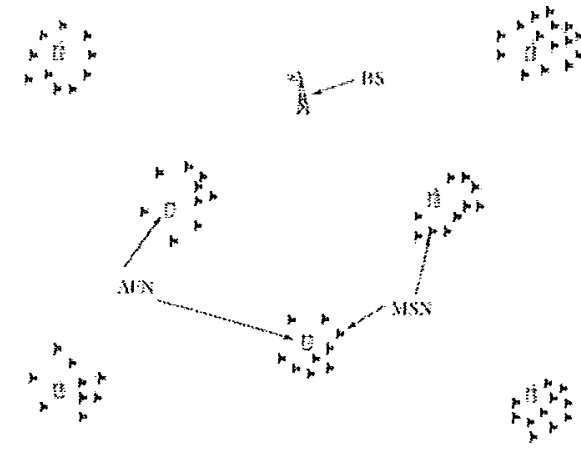

(a) Physical topology.

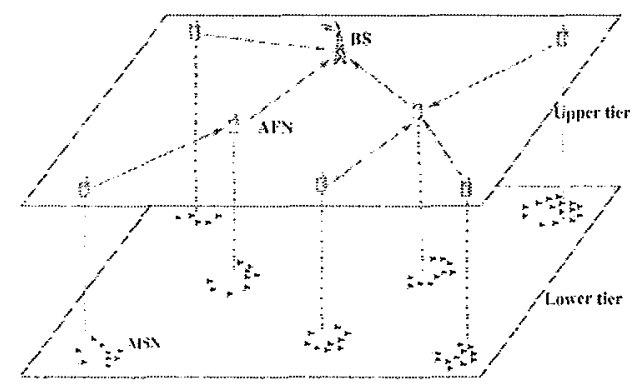

(b) Hierarchical view.

Figure 2.4: Reference Architecture for a Two-Tiered Wireless Sensor Network.

The study in Reference [32] considers the joint problem of energy provisioning for a two-tiered wireless sensor network. The two-tiered wireless sensor network architecture as shown in Figure 2.4 is described and power consumption and power control in this architecture is reviewed. The problem of deploying relay nodes, given a set of existing nodes, is studied. The reference shows for a given network with initial energy, how additional energy should be allocated to either existing or new locations.

An attempt to maximize network lifetime by arranging basestations and relay nodes positions in a two-tiered Wireless Sensor Network is done in Reference [33]. In this architecture sensors are in the lower tier of the network. They are deployed as clusters and capture, encode and transmit data to an application node. The application node then processes the data and creates a comprehensive local-view and forwards it to a basestation. Both application node and basestation are in the higher tier of the network. They are also battery-powered and energy-constrained. The optimal location is only determined for the simple case where only single-hop routing is allowed. The more 


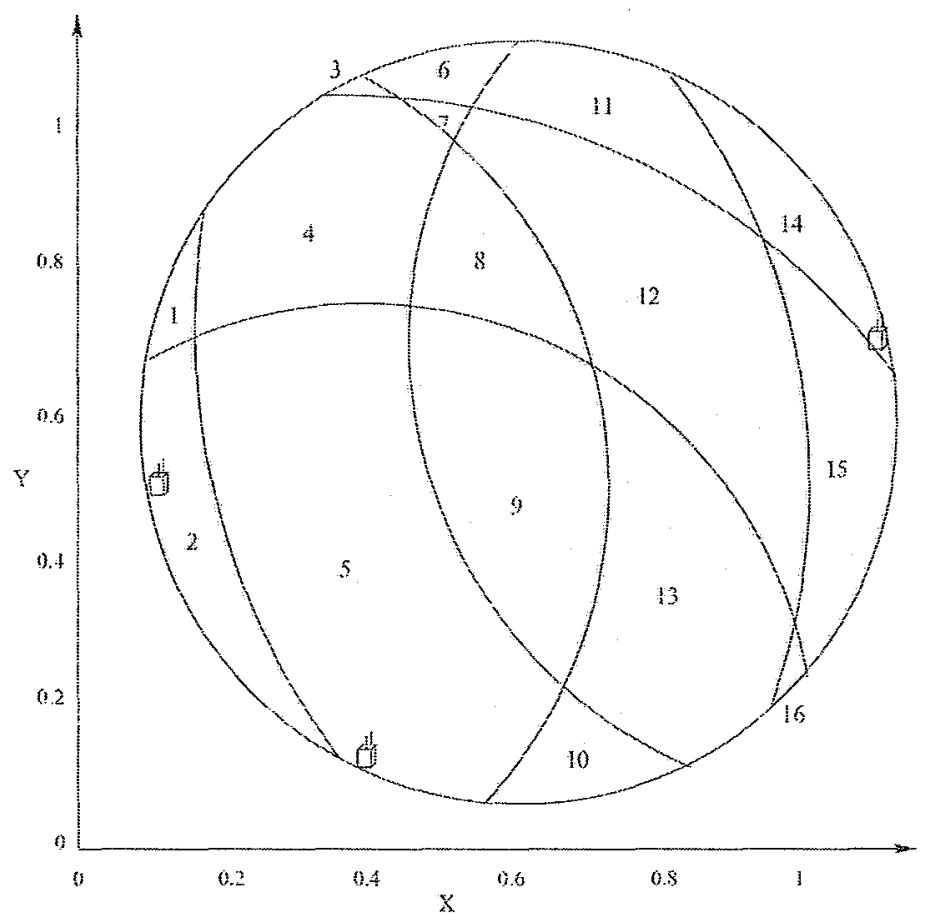

Figure 2.5: The disk is divided into 16 subareas for the 3-node example.

difficult problem involving multi-hop routing is not addressed.

Placing basestations so that the network flow is proportionally maximized subject to link capacity is studied in [34]. It is showen even though it is possible to find optimal solutions for a special network topology (e.g., grid); the base station placement problem for an arbitrary network is NP-complete.

A $(1-\epsilon)$ optimal approximation algorithm to the basestation placement problem is proposed in Reference [35]. This is done by constructing a finite search space of physical points.

A network model for basestation placement in a battery powered network problem is presented in [6]. This work includes an approximation algorithm 
M.A.Sc. Thesis - Sayed-Ali Shariatmadari McMaster - Electrical Engineering

that can guarantee $(1-\epsilon)$ optimal network lifetime performance for base station placement problem with any desired error bound $\epsilon>0$. The algorithm first finds the smallest disk that contains all of the nodes. For each node lower and upper cost bound is computed and based on these bounds a sequence of costs is defined. Then sequences of circles are drawn centered at each node with increasing radius corresponding to cost series defined for that node. The intersection of these circles creates subareas in the disk that contains all of the nodes. Then an optimization problem that is linear is solved to find the best subarea for the basestation that maximizes network lifetime. The basestation can be placed anywhere in the selected subarea. A sample of a disk divided into subareas is shown in Figure 2.5. The performance of proposed algorithm is better than the one proposed in Reference [35]. It is also proved that the proposed base station placement algorithm is $(1-\epsilon)$ optimal. But the assumption is that there is no bound on the transmit power. This work is extended in [36]. The first extension is the case where the transmit power at each sensor node is upper bounded. It is showed that the proposed algorithm can be extended without much difficulty. The second extension is to show that how the proposed algorithm can be extended for multiple basestations. This is done by narrowing down the search space for each basestation location into a finite search space while the performance of the algorithm is guaranteed. 


\section{Chapter 3}

\section{Solar Powered Networks}

\subsection{Introduction}

Having a battery powered wireless embedded system (such as sensor nodes) operate in an energy sustainable fashion is a key challenge and a lot of research effort has been devoted to energy optimization of such systems. Energy harvesting from the environment, in particular solar power based, has increasingly been used as a practical technique to supplement battery supplies. In this chapter we survey different methods and hardware designed to make harvesting of the energy from the environment more efficient. 
M.A.Sc. Thesis - Sayed-Ali Shariatmadari McMaster - Electrical Engineering

\subsection{Solar Radiation Data}

Theoretically, it is impossible to know in advance how much solar energy would be received at the solar panel of a sensor. The motion of the earth creates daily and yearly cyclic behavior in solar insolation, which are deterministic, but weather conditions such as air humidity and clouds add randomness to the amount of harvested energy and are mainly modeled using stochastic processes. However, it can be assumed that weather conditions have a cyclostationary property and past solar irradiation data can be used. For this purpose, we can use public meteorological data and depending on the site specific situation some conversion of meteorological data are required [37].

\subsection{Hardware Design of Solar Sensors}

Several technologies have been developed to extract different types of energy from the environment. Also several solar energy-harvesting sensors have been developed. In Reference [38] an upper bound for conversion of solar energy is investigated. Important factors such as components, design choices, tradeoffs that are involved in designing of solar powered sensors are discussed in Reference [39]. An implementation by means of super capacitors and lithium rechargeable batteries is introduced in [40]. Several important aspects of design and architecture of micro-solar power system sensors are investigated in [41]. In [42] a method to harvest energy from other nodes is studied by building a network that consists of fixed and mobile nodes. Mobile nodes always move and search for energy, recharge themselves and bring energy 
M.A.Sc. Thesis - Sayed-Ali Shariatmadari McMaster - Electrical Engineering

to non-movable nodes. Design principles for a long duration solar powered wireless sensor network is discussed in [43] and data from an actual implementation is presented. It uses a DC-DC converter and simple NiMH battery technology.

\subsection{Energy Aware Design Challenges}

The design goal in a network that is powered with batteries is to minimize the power consumption of network nodes [44] or to maximize the network lifetime [45]. An energy-harvesting node is defined as a system which gets some or all of its energy from the environment. A vital difference between this kind of energy and that from a battery is that the amount of energy that can be harvested from the environment can be unlimited. A harvesting network can be built by using energy-harvesting nodes [9].

At first glance it seems that this is also true for energy harvesting networks and tasks must be allocated to nodes with higher residual battery, because these nodes have harvested more energy than others. But it has been shown in References [46], [47] and [39] that making power consumption decisions that are based on environment conditions have better results than otherwise. There are two main reasons. The first is that the workload of a node with recharging opportunity may not match the pattern that the node harvests energy from the environment. If more work is assigned to nodes with higher battery size, over time this may reduce the battery reserves of those nodes. The second reason is that to be able to decide how much energy can be consumed and saved, the 
M.A.Sc. Thesis - Sayed-Ali Shariatmadari McMaster - Electrical Engineering

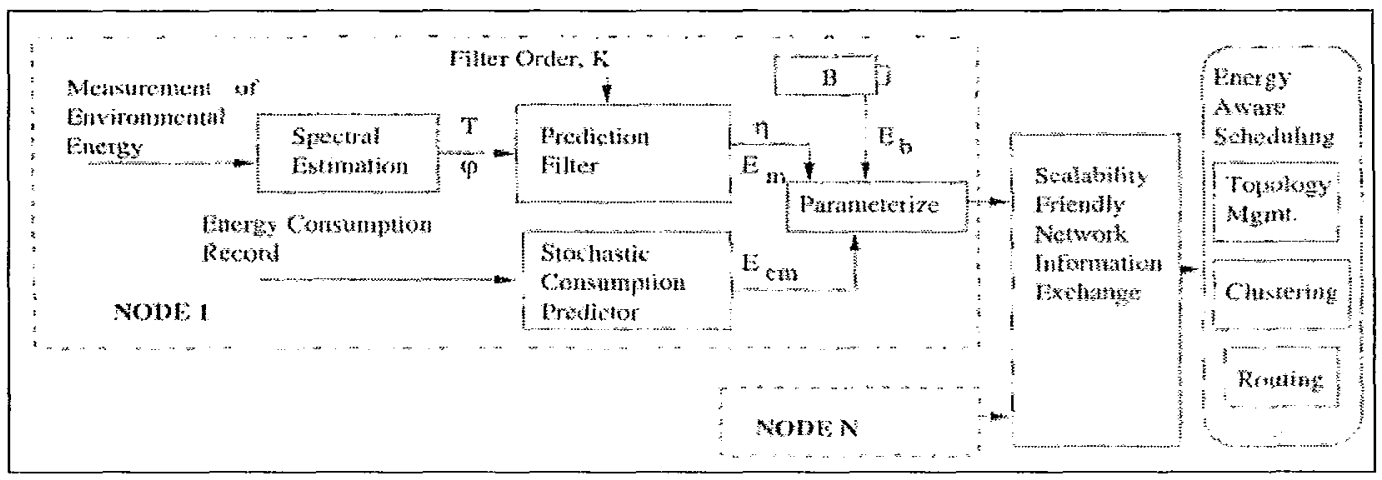

Figure 3.1: Interactions Between Various EEHF Algorithms

environmental energy must be known. For example in a wireless sensor network, nodes with more available solar energy must use more transmit power than other nodes and even relay traffic for them. Therefore energy harvesting networks must utilize energy based on environmental energy, not the residual battery content of the nodes.

In [46] the spatiotemporal property of the available energy in a network, i.e., the harvesting problem, is discussed. A distributed framework referred to as. environmental energy harvesting framework (EEHF) is the first part of the solution that is provided to solve the problem. This framework is used for adaptively learning the energy properties of the environment and the renewal opportunity at each node and to make this information available for use in other tasks. The EEHF block diagram is presented in Figure 3.1.

Power management in energy harvesting networks is discussed in Reference [9]. The paper discusses the conditions for energy-neutral operation in detail and models the variation of energy sources and energy consumption. Energy-Neutral operation is achieved by consuming energy such that the energy used is always less than the energy harvested. Both the spatiotemporal 
M.A.Sc. Thesis - Sayed-Ali Shariatmadari McMaster - Electrical Engineering

profile of the available energy and the way of usage of that energy is very important to guarantee the maximum performance of the network.

In Reference [9] some practical methods are developed which an energy harvesting system can use to predict the expected energy that can be harvested. The model is motivated by leaky-bucket Internet traffic models. However, there is a difference between this model and the leaky-bucket model. In Internet traffic policing a limit is only needed on the maximum traffic bursts. But in harvesting energy, bounding both the maximum and minimum energy outputs is required. In [48] different solar energy prediction algorithms are compared. These algorithms give estimates of available energy over future time intervals.

An algorithm called Uniform Sensing Protocol is proposed in [49], which uses ANSWER (AutoNomouS netWorked sEnsoR system) [50] as its structure. In ANSWER, nodes possess certain mathematical capabilities and a clustering system is used to organize the micro-sensors. Uniform Sensing Protocol uses this structure to determine routing. Each sensor node must first calculate its energy budget at the beginning of each day and night cycle.

In the Uniform Sensing Protocol, the sensor nodes first calculate their energy budget at the beginning of each day and night cycle and based on that, the active time for the sensor is calculated. The protocol uses a Gaussian distribution function to calculate the probabilities of possible needed activities. If any activity is requested the node increases the active time period, otherwise active time remains unchanged. 
M.A.Sc. Thesis - Sayed-Ali Shariatmadari McMaster - Electrical Engineering

\subsection{Solar Based Routing Algorithms}

Routing in wireless sensor networks has to take into account the very limited resources of the nodes. We will review some of the solar aware proposed protocols. These protocols follow the idea that as far as it is possible, tasks should be carried out using the nodes that have ability to harvest environment energy rather than consuming energy from node batteries which do not have any recharging opportunity. Two protocols that perform solar aware routing decisions are proposed and evaluated in [47]. These protocols are based on directed diffusion.

In direct diffusion the node that is interested in data is called sink and is looking for a sensor that has that data. Interests for data are attribute-value pairs. The source is the sensor that can deliver that data and potential sources are called gradients. Events, which are the source data, are sent to the sink via gradients. The simplest form of the protocol is sending information to the sink via broadcasting the data. Then the sink sends another interest message and this time by using the responses from the first stage, the best path is chosen. This path is chosen based on some factors like earliest response or lowest loss rate, and the data will only send to one neighbor. In direct diffusion at least adjacent nodes are required to be distinguished by identifiers.

The first solar aware protocol is based on local interaction with neighboring nodes. It is a simplified version of the direct diffusion protocol. In this protocol gradients also contain solar state of the neighbors. The nodes with solar power use the option to send to a solar powered neighbor. When a node changes its solar status, it should inform the upstream nodes. The second protocol extends 
M.A.Sc. Thesis - Sayed-Ali Shariatmadari McMaster - Electrical Engineering

standard directed diffusion by adding several fields to the standard directed diffusion headers protocol. The original directed diffusion scheme does not prevent loops to occur but detects and removes them. But this protocol has a scheme that prevents loops in the first place. The result shows that first protocol works better with smaller networks and the second one for the larger network. Both of the protocols have significant energy saving effects.

A QoS-aware geographic routing algorithm that is based on a solar energy is proposed in [51]. The proposed algorithm tries to determine the topological knowledge range (KR) and select an appropriate route. The larger the knowledge range is the more likely to produce a near optimal path. On the other hand, gathering and maintaining more topological information requires more energy. An algorithm named Adaptive toPOLogical KR aLgOrithm (APOLLO) is proposed. By exploiting an estimation of harvested energy in a period of time, it periodically and locally determines the topological knowledge range of each node. The second algorithm named PrIority-based path Selection Algorithm (PISA) runs on each node to find a route by using the knowledge range of the node, while it tries to meet different objectives such as path delay, energy consumption and reliability.

\subsection{IEEE 802.11 Mesh Networks}

WLAN mesh networks are currently being deployed for outdoor wireless coverage in many areas to create Wi-Fi coverage zones. Providing continuous electrical power for all of these nodes is a major challenge in these mesh networks. Using solar or wind power as an energy source for some of these nodes 


\section{M.A.Sc. Thesis - Sayed-Ali Shariatmadari McMaster - Electrical Engineering}

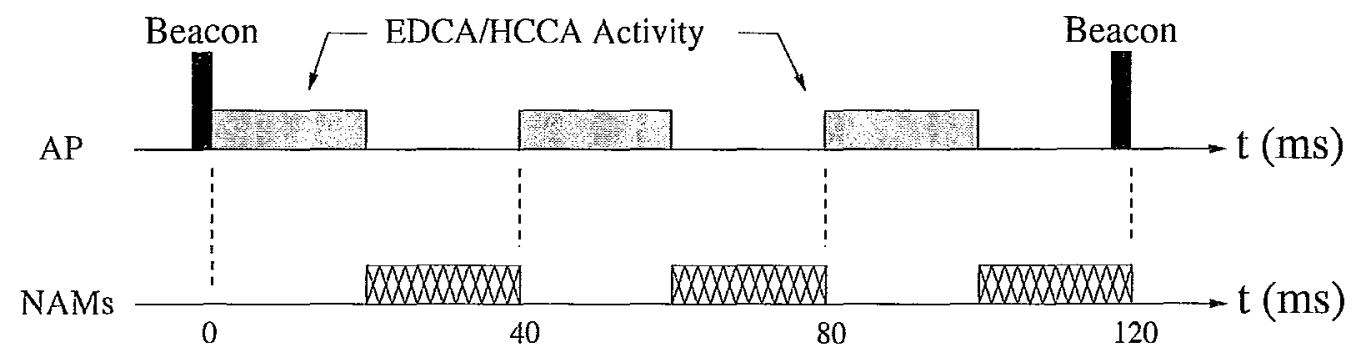

Figure 3.2: Best-effort mesh AP power saving with movable boundary.

is an alternative to a fixed power connection. The SolarMESH network is an example that uses this approach in an operational testbed [52].

A resource allocation problem in solar powered WLAN mesh nodes is studied in Reference [8]. It is shown that the use of power saving methods in mesh nodes significant reduces the node cost. Some outage control strategies are also presented that can prevent outage of a mesh node. Legacy IEEE 802.11 only provides power saving modes for nodes and does not have any mechanism for placing access points into a power saving mode. IEEE 802.11e has a power saving mechanism that includes both contention and polling based options. Two variations of IEEE 802.11 that support power saving access points are proposed in References [53] and [54]. Adaptive modification of access point sleeping schedules based on current load conditions is proposed in Reference [53] and the method proposed in Reference [54] is a power saving WLAN mesh architecture based on the IEEE 802.11e.

In Reference [54] a power saving AP broadcasts a network allocation map (NAM) in its beacon, which includes the information of periods of time within the superframe that the AP is unavailable. An example of this type of activity for a single inter-beacon period is shown in Figure 3.2. The upper timeline 
M.A.Sc. Thesis - Sayed-Ali Shariatmadari McMaster - Electrical Engineering

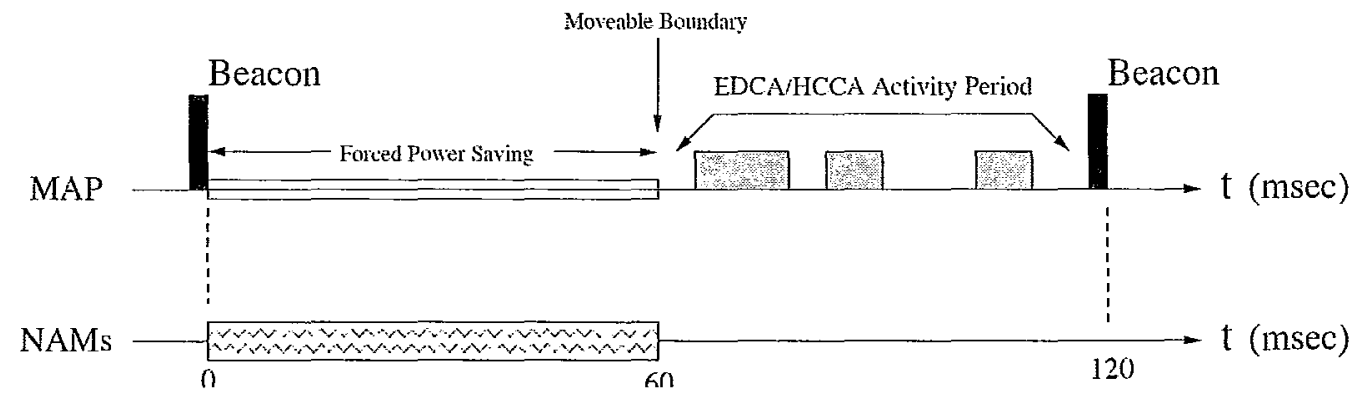

Figure 3.3: Forced mesh AP power saving (FPS) with $50 \%$ offered capacity.

shows the activity of the channel and the lower timeline shows the NAMs. It is also possible to force a maximum level of activity for an AP. This is referred to as Forced Power Saving (FPS). Figure 3.3 shows an example of an AP that is using FPS. In this example the AP advertises a NAM restricting its activity to a maximum of $50 \%$ of the inter-beacon interval. One of the most important uses of this capability is the ability to develop outage control algorithms [8].

A simplified version of a solar powered WLAN mesh node is shown in Figure 3.4. The solar panel and battery are connected to the AP through a charge controller which performs functions such as battery over/undercharge protection. We can define an energy flow model for this configuration [7]. In each time epoch $t, E_{i}(t)$ is the available energy for Node $i$ and $t$ ranges over the entire set of solar irradiation samples and the energy stored at each sensor's battery must satisfy the following recursion

$$
B_{i}(t)=\min \left(\max \left(B_{i}(t-1)+E_{i}(t)-L_{i}(t), B_{\text {outage }}\right), B_{\max }(i)\right) .
$$

In this equation, $E_{i}(t)$ is the maximum solar insolation available at Node $i$ during the current time epoch. This equation is an energy recursion that computes 
M.A.Sc. Thesis - Sayed-Ali Shariatmadari McMaster - Electrical Engineering

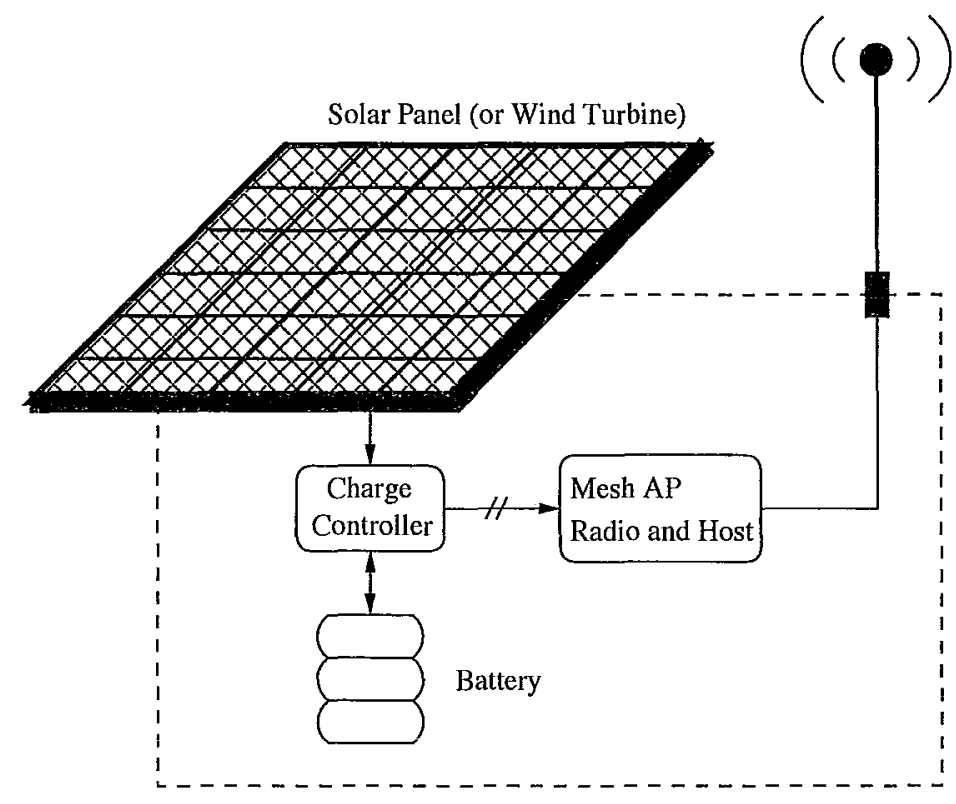

Figure 3.4: Solar Powered WLAN Mesh Node

at each time epoch, the amount of energy that a sensor node obtains as $E_{i}(t)$ and the amount of energy that is utilized as $L_{i}(t)$. The amount of energy stored in the battery cannot exceed $B_{\max }(i)$ and also cannot be less than $B_{\text {outage. }}$

The problem of cost-optimal placement of the energy sustainable nodes in these type of WLAN mesh networks is studied in Reference [55]. At first a cost model is introduced that considers the provisioning required to operate the solar or wind powered nodes with a known outage criterion. Then this problem is formulated as Mixed Integer Quadratic Problem and a branch and bound method is used to find the solutions for node positioning. A methodology for the provisioning of energy sustainable mesh nodes was given in [56]. This was applied to IEEE 802.11 mesh networks operating using solar power, and is the methodology used for resource provisioning in this thesis. Over-provision of nodes when using energy aware routing is discussed in Reference [57]. In this 
M.A.Sc. Thesis - Sayed-Ali Shariatmadari McMaster - Electrical Engineering work energy aware routing is also incorporated in resource assignment and a genetic algorithm (GA) has been developed for this purpose. 


\section{Chapter 4}

\section{Problem Definition}

\subsection{Introduction}

In this chapter we define and formulate the problem. Consider a wireless network of sensor nodes where the nodes are powered by solar energy. Each node communicates with the wired network through one or more sensor node air hops to an appropriate basestation. We assume a tiered system where all the basestations have infrastructure connectivity, and hence a given sensor node is free to communicate using any choice of basestation. In this thesis, we use basestation placement in order to optimize the sustainable energy performance of the sensor nodes. The objective of the problem is therefore to locate and minimize the number of deployed basestations needed to support the sensor nodes so that they are energy sustainable, i.e., outage-free.

We assume that the candidate basestation locations are known in advance. To obtain an efficient basestation placement, basestation density must in general be higher in regions where there are sensor nodes with low energy renewal 


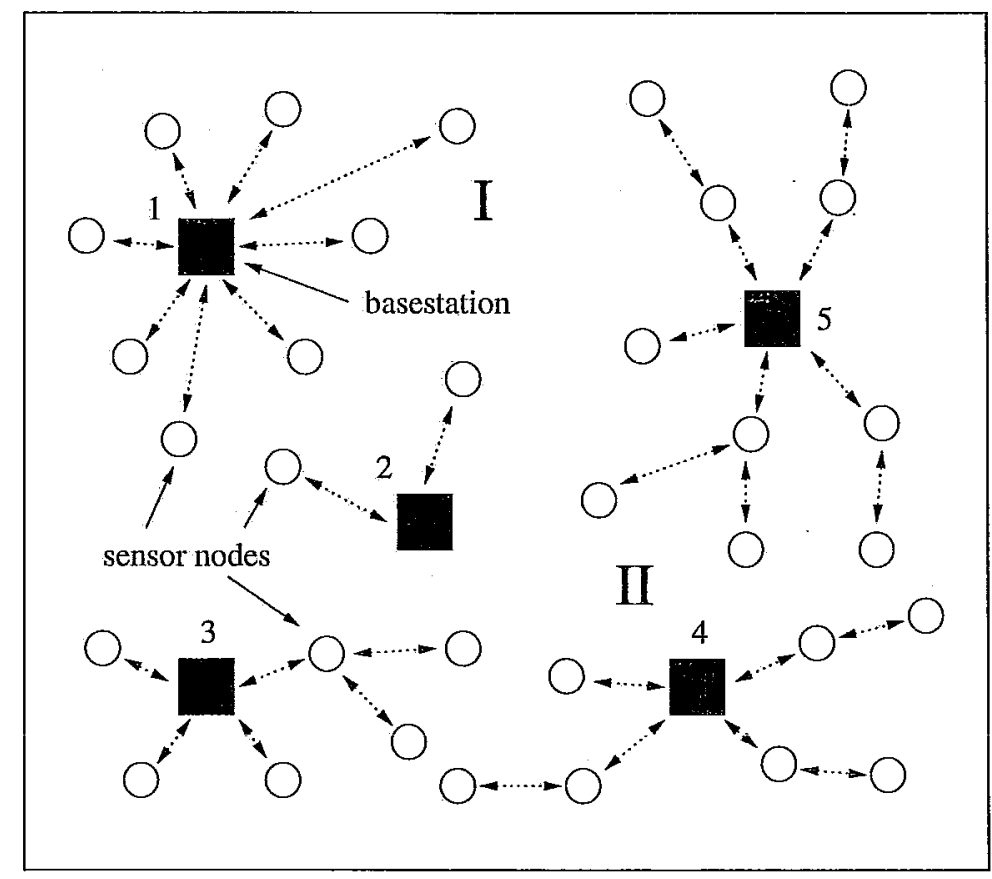

Figure 4.1: Basestations and Solar Powered Sensor Nodes. Region I has a lower solar factor than Region II. The deployed basestation density is lower in Region II.

rates and/or high node utilizations. This is needed to reduce the level of multihop communication that is needed to maintain full connectivity. Otherwise sensor nodes in those regions may suffer outage due to insufficient solar energy reserves. In this model, the communication range of the sensor nodes is bounded by defining a limit on transmission power, which induces a graph where the edges are potential links between sensor nodes.

The problem formulation also considers the effects of the spatial distribution of solar insolation. This is a consideration that is often neglected, but is an important issue in practical solar powered mesh nodes [7]. Although the methodology in this thesis applies to generalized per unit area solar insolation 
M.A.Sc. Thesis - Sayed-Ali Shariatmadari McMaster - Electrical Engineering

renewal rates, for each Node $i$, we define a solar factor, $\alpha_{i}(t) \in(0,1)$, which gives the fraction of available solar energy due to shadowing and positional restrictions compared with an optimally positioned panel at that location. We assume that these insolation factors are known to the basestation deployment algorithm based on information collected during a site survey when the sensor nodes are placed.

Figure 4.1 shows an illustrative example. The two shaded areas represent regions with different solar insolation factors. Deployed basestations are shown as black squares and sensor nodes are shown as circles. The dotted arrows show the wireless hops used for routing. In the figure, Region I has a lower solar insolation factor than in Region II. For this reason, sensor nodes in Region II can support levels of multi-hop relaying that lead to a decreased basestation deployment density in that region. When this is the case, this can have a strong effect on the optimum number and locations of the basestations needed to support energy sustainable node operation.

\subsection{Problem Formulation and Lower Bound}

Each sensor node is represented by an integer in the set $\mathcal{N}=\{1,2, \ldots, N\}$, and each potential basestation location is indexed by an integer in the set $\mathcal{B}=\{N+1, N+2, \ldots, N+B\}$. Since sensor traffic can be routed through any basestation, the flows between a sensor node and the infrastructure can be represented by a single value. Accordingly, $\mathcal{F}$ is defined as the set of all nodes in $\mathcal{N}$ plus one additional node representing the basestations. Thus the cardinality of $\mathcal{F}$ is equal to $N+1$. Table 4.1 lists all notations used in this thesis. 
M.A.Sc. Thesis - Sayed-Ali Shariatmadari McMaster - Electrical Engineering

\begin{tabular}{|c|l|}
\hline$T$ & Number of time epochs \\
$\mathcal{B}$ & Number of sensor nodes \\
$\mathcal{N}$ & Number of placement for basestations \\
$\mathcal{B}$ & Set of sensors $=\{1,2, \ldots, N\}$ \\
$\mathcal{S}$ & Set of placement for basestations $=\{N+1, N+2, \ldots, N+B\}$ \\
$\mathcal{F}$ & Set of all nodes in $\mathcal{N}$ and base stations in $\mathcal{B}$ \\
$\mathcal{A}$ & Set of sources and destination of flows $=\{1,2, \ldots, N+1\}$ \\
$G(\mathcal{S}, \mathcal{A})$ & Set of all links that exist in network \\
$g_{s d}(t)$ & Network topology graph \\
$\gamma_{i j}^{s d}(t)$ & Flow volume that is generated in node $s$ at each time epoch and is \\
$p_{t, i j}, p_{r, i}$ & destined to $d$ \\
$p_{i d l l}$ & Part of the flow $g_{s d}(t)$ that goes from node $i$ to node $j$ \\
$L_{i}(t)$ & Energy consumption factors for transmitting and receiving data \\
$B_{i}(t)$ & Total Load of a sensor $i$ at each time slot \\
$B_{\text {max }}(i)$ & Residual battery energy of sensor $i$ at time $t$ \\
$B_{\text {outage }}$ & Maximum Battery capacity \\
$E_{i}(t)$ & Maximum allowed depth of battery discharge \\
$\alpha_{i}(t)$ & Solar insolation at node $i$ at time $t$ \\
$C_{i j}$ & Solar insolation attenuation coefficient in node $i$ at time $t$ \\
$\mathcal{R}_{i j}$ & Capacity of link between $i$ to $j$ \\
$d_{i j}$ & Relaying table \\
$\beta_{1}, \beta_{2}, m$ & Distance between node $i$ and node $j$ \\
$w_{1}, w_{2}, w_{3}$ & Power consumption and path loss coefficients for receiver and \\
\hline & Weight assigned to each criteria of the objective \\
\hline
\end{tabular}

Table 4.1: Notations

We define $\lambda_{i}$ to be a binary decision variable that determines whether a basestation is placed at a given location in $\mathcal{B}$. The network is modeled as a graph $G(\mathcal{S}, \mathcal{A})$ where $\mathcal{S}$ consists of all of the nodes in $\mathcal{B}$ and $\mathcal{N}$, and $\mathcal{A}$ is the set of all links $(i, j)$ where $i, j \in \mathcal{S} . e_{i j}$ is also defined to remove the links between nodes that do not have any mutual connection, which are known in 
M.A.Sc. Thesis - Sayed-Ali Shariatmadari McMaster - Electrical Engineering

advance and are defined as follows.

$$
e_{i j}= \begin{cases}1 & (i, j) \in \mathcal{A} \\ 0 & \text { ow. }\end{cases}
$$

We now consider the bandwidth flow and sensor node power consumption. To account for traffic flow continuity, we assume that $s$ is the source and $d$ is the destination of a flow with volume $g_{s d}(t)$. Therefore, $s$ and $d$ are in $\mathcal{F}$ and part of that flow that is passed from Node $i$ to $j$ can be written as $\gamma_{i j}^{s d}(t)$. The flow continuity for any node $i \in \mathcal{N}$ can be written as,

$$
\sum_{j \in \mathcal{N}}\left(e_{i j} \gamma_{i j}^{s d}(t)-e_{j i} \gamma_{j i}^{s d}(t)\right)+\sum_{j \in \mathcal{B}} \lambda_{j}\left(e_{i j} \gamma_{i j}^{s d}(t)-e_{j i} \gamma_{j i}^{s d}(t)\right)= \begin{cases}g_{s d}(t) & i=s \\ -g_{s d}(t) & i=d \\ 0 & \text { ow. }\end{cases}
$$

The first term on the left hand side of Equation (4.2) is the fraction of the flow that a sensor node sends to others for relaying. The second term is that sent from a sensor to a basestation. The third is the flow that is received by the sensor from other sensors and the fourth is that received by the sensor from a basestation. $\lambda_{i} \gamma_{i j}^{s d}(t)$ means that traffic passes through Basestation $i$, if that basestation location is used. The left hand side is positive and equal to $g_{s d}(t)$ if this node is the source of traffic and is equal to $-g_{s d}(t)$ if it is the destination, otherwise it must be zero because of flow continuity. Because the basestation flows are modeled as a single node, flow continuity for all basestations must 
M.A.Sc. Thesis - Sayed-Ali Shariatmadari McMaster - Electrical Engineering

be written as one equation that is similar to Equation (4.2), i.e.,

$$
\sum_{i \in \mathcal{B}} \sum_{j \in \mathcal{N}} \lambda_{i}\left(e_{i j} \gamma_{i j}^{s d}(t)-e_{j i} \gamma_{j i}^{s d}(t)\right)= \begin{cases}g_{s d}(t) & s \in \mathcal{B} \\ -g_{s d}(t) & d \in \mathcal{B} \\ 0 & \text { ow. }\end{cases}
$$

For each Basestation $i$, the first term in Equation (4.3) is the traffic that the basestation sends to sensor nodes, and the second term is the traffic that is received from sensor nodes. We also include a link capacity constraint that is written as

$$
0 \leq \sum_{s, d} e_{i j} \gamma_{i j}^{s d}(t) \leq C_{i j}
$$

The result of the summation is the total traffic from all of the flows that pass through Node $i$ to Node $j$. By using the above definitions, the total load at each node $i \in \mathcal{N}$ is

$$
\begin{gathered}
L_{i}(t)=\sum_{s, d} \sum_{j \in \mathcal{N}, \mathcal{B}}\left(e_{i j} p_{t, i j} \gamma_{i j}^{s d}(t)+e_{j i} p_{r, i} \gamma_{j i}^{s d}(t)\right)+p_{i d l e} \sum_{j \in \mathcal{N}, \mathcal{B}}\left(e_{i j} I_{i j}(t)+e_{j i} I_{j i}(t)\right) \\
I_{i j}(t)=\frac{C_{i j}-\sum_{s, d} \gamma_{i j}^{s d}(t)}{C_{i j}}
\end{gathered}
$$

This equation is similar to the traffic flow equation. The first part of Equation (4.5) is the total energy that is consumed for transmitting between nodes and basestations. The second part is the power consumption of the sensor when it is idle. The normalization of link utilization that gives the fraction of time that links are idle is written in Equation (4.6). The $p_{t, i j}$ and $p_{r, i}$ coefficients respectively, describe the amount of energy that a sensor uses for transmitting 
M.A.Sc. Thesis - Sayed-Ali Shariatmadari McMaster - Electrical Engineering

or receiving one unit of traffic. $p_{r, i}$ is considered constant and $p_{t, i j}$ is given by

$$
p_{t, i j}=\beta_{1}+\beta_{2} \cdot d_{i j}^{m}
$$

where $\beta_{1}$ is a distance independent term and $\beta_{2}$ is a distance dependent one, $m$ is the path loss coefficient, with $2 \leq m \leq 4 . d_{i j}$ is the distance between two nodes [6]. Note that although we assume an exponential path loss, any propagation model can be incorporated into the computation.

Solar powered systems are modeled in discrete time, and hence the lifetime of the network is divided into time epochs, which are usually 1-hour in duration [7]. In each time epoch $t, E_{i}(t)$ is the available energy for Sensor $i$ and $t$ ranges over the entire set of solar irradiation samples. The target network lifetime is specified in advance, and the energy stored at each sensor's battery must satisfy the following recursion [7],

$$
B_{i}(t)=\min \left(\max \left(B_{i}(t-1)+\alpha_{i}(t) E_{i}(t)-L_{i}(t), B_{\text {outage }}\right), B_{\max }(i)\right) .
$$

In this equation, $E_{i}(t)$ is the maximum solar insolation available at Node $i$ during the current time epoch. $\alpha_{i}(t)$ is the solar factor which discounts this value as discussed previously, and may be time-varying. This equation is an energy recursion that computes at each time epoch, the amount of energy that a sensor node obtains is $\alpha_{i}(t) E_{i}(t)$ and the amount of energy that is utilized is $L_{i}(t)$. The amount of energy stored in the battery can not exceed $B_{\max }(i)$ and also cannot be less than $B_{\text {outage [7]. }}$

Equations (4.2) and (4.3) are non-linear, i.e., $\lambda_{k}$ is a binary variable that 
M.A.Sc. Thesis - Sayed-Ali Shariatmadari McMaster - Electrical Engineering

is multiplied by another, $\gamma_{i j}^{\text {sd }}(t)$. This term is linearized by defining a new variable $v_{i j, k}^{s d}$ with the following conditions [58].

$$
\begin{gathered}
0 \leq v_{i j, k}^{s d} \leq \gamma_{i j}^{s d}(t), \\
\gamma_{i j}^{s d}(t)-C_{i j}\left(1-\lambda_{k}\right) \leq v_{i j, k}^{s d} \leq \lambda_{k} C_{i j} .
\end{gathered}
$$

Now when $\lambda_{k}=0$, then $0 \leq v_{i j, k}^{s d} \leq 0$ and $v_{i j, k}^{s d}$ is forced to be zero and when $\lambda_{k}=1$ then $\gamma_{i j}^{s d}(t) \leq v_{i j, k}^{s d} \leq \gamma_{i j}^{s d}(t)$ and $v_{i j, k}^{s d}$ is forced to be equal to $\gamma_{i j}^{s d}(t)$. Index $(i j, k)$ means that the link is between $i$ and $j$ and $k$ is a basestation.

In Equation (4.8), the minimum of $B_{i}(t)$ is $B_{\text {outage }}$ and its maximum is $B_{\max }(i)$. In some situations however, the load is more than the harvested energy plus energy stored in battery. Another possible situation happens when the harvested energy overfills the capacity of the battery. To linearize this equation, a new slack variable, $r_{i}(t)$, is defined such that,

$$
\begin{gathered}
B_{\text {outage }} \leq B_{i}(t) \leq B_{\max }(i) \\
B_{i}(t)+r_{i}(t)=B_{i}(t-1)+\alpha_{i}(t) E_{i}(t)-L_{i}(t) \\
r_{i}(t) \geq 0
\end{gathered}
$$

$r_{i}(t)$ must be minimized in order that this formulation be true. Therefore it is added to the problem as a new term in the objective function. In addition, $r_{i}(t)$ must be positive, to constrain the sensor node to never go into outage.

Another concern in the formulation of the problem is dealing with routing loops, which is common in energy harvesting problems. For example consider a sensor network with 10 nodes that is supposed to work for 10 hours. Assume 
M.A.Sc. Thesis - Sayed-Ali Shariatmadari McMaster - Electrical Engineering

that 3 basestations are enough for non-interruptible service. It is possible in some time epochs that some nodes have extra energy stored in their batteries. If there is flow that passes through these nodes, they can form a routing loop. This happens because these loops do not contradict the flow continuity (Eq. (4.2) and (4.3)) and energy flow model (Eq. (4.8)). Therefore the final data routing result may contain incorrect results. These loops can be eliminated from routings by minimizing the sum of total routing of data over all time epochs.

The above definitions result in a multi-criterion linear mixed-integer optimization problem that can be used to find a lower bound on the number of deployed basestations. It is worth mentioning that nature of this specific problem automatically minimized the slack variable $r_{t}(i)$. The slack variable stores the amount of solar energy that is harvested by a node, but is wasted because battery is fully charged and it must be minimized. But when the problem is minimizing the number of basestations in the network, this variable is minimized automatically. This happens because a higher value of slack variable means less energy is stored in the battery of the nodes, therefore less energy is available for data transmission and this means placing more basestations which contradicts the main objective of the problem. Thus in this specific situation minimizing slack variable can be removed from the objective function. This is not always true, for example the algorithms that will be discussed later in this chapter do not follow this exception.

The problem can be formulated by assigning different weights $\left(w_{1}, w_{2}\right.$ and $\left.w_{3}\right)$ to different criteria, resulting in the following final formulation, 
M.A.Sc. Thesis - Sayed-Ali Shariatmadari McMaster - Electrical Engineering

$$
\text { minimize } w_{1} \sum_{i \in \mathcal{B}} \lambda_{i}+w_{2} \sum_{\substack{i \in \mathcal{N} \\ t \in\{1,2, \ldots, T\}}} r_{i}(t)+w_{3} \sum_{\substack{s, d \in \mathcal{F} \\(i, j) \in \mathcal{A} \\ t \in\{1,2, \ldots, T\}}}^{s d d}(t)
$$

subject to

$$
\begin{gathered}
\lambda_{i} \in\{0,1\} \\
0 \leq \sum_{s, d} e_{i j} \gamma_{i j}^{s d}(t) \leq C_{i j} \\
\sum_{j \in \mathcal{N}}\left(e_{i j} \gamma_{i j}^{s d}(t)-e_{j i} \gamma_{j i}^{s d}(t)\right)+\sum_{j \in \mathcal{B}} \lambda_{j}\left(e_{i j} \gamma_{i j}^{s d}(t)-e_{j i} \gamma_{j i}^{s d}(t)\right)= \begin{cases}g_{s d}(t) & i=s \\
-g_{s d}(t) & i=d \\
0 & \text { ow. }\end{cases} \\
\sum_{i \in \mathcal{B}} \sum_{j \in \mathcal{N}} \lambda_{i}\left(e_{i j} \gamma_{i j}^{s d}(t)-e_{j i} \gamma_{j i}^{s d}(t)\right)= \begin{cases}g_{s d}(t) & s \in \mathcal{B} \\
-g_{s d}(t) & d \in \mathcal{B} \\
0 & \text { ow. }\end{cases} \\
L_{i}(t)=\sum_{s, d} \sum_{j \in \mathcal{N}, \mathcal{B}}\left(e_{i j} p_{t, i j} \gamma_{i j}^{s d}(t)+e_{j i} p_{r, i} \gamma_{j i}^{s d}(t)\right)+p_{i d l e} \sum_{j \in \mathcal{N}, \mathcal{B}}\left(e_{i j} I_{i j}(t)+e_{j i} I_{j i}(t)\right) \\
I_{i j}(t)=\frac{C_{i j}-\sum_{s, d} \gamma_{i j}^{s d}(t)}{C_{i j}} \\
B_{\text {outage }} \leq B_{i}(t) \leq B_{\max }(i)
\end{gathered}
$$


M.A.Sc. Thesis - Sayed-Ali Shariatmadari McMaster - Electrical Engineering

\subsection{Energy Aware Basestation Placement}

The optimization bound formulated in Equation (4.12) is a linear mixed-integer problem. It is proved that this is NP-hard, and can only be solved for small problem sizes [12]. In addition, the bound does not in general result in a causal basestation deployment solution, since it uses knowledge of the full solar insolation trace in its computation. For these reasons we propose a simple and efficient practical algorithm. The algorithm is first described, and then a method for generating an initial solution is discussed based on an Iterated Local Search (ILS) procedure [59].

The proposed algorithm is shown in Algorithm 4.1. The algorithm starts with an initial solution and then solves the problem for each time epoch using the solar insolation inputs from the previous step (Line 4). The algorithm stores and updates a table that tracks the frequency with which the node relays traffic on behalf of other nodes. If a node runs into outage during this process, it uses this information to determine new basestation activations (Line 9 and Line 10). By activating a basestation that these specific nodes require for relaying, the transit traffic that passes through the failed node can be decreased significantly.

As mentioned above, the objective of the Lower Bound problem is

$$
\text { minimize } \quad w_{1} \sum_{i \in \mathcal{B}} \lambda_{i}+w_{2} \sum_{\substack{i \in \mathcal{N} \\ t \in\{1,2, \ldots, T\}}} r_{i}(t)+w_{3} \sum_{\substack{s, d \in \mathcal{F} \\(i, j) \in \mathcal{A} \\ t \in\{1,2, \ldots, T\}}} \gamma_{i j}^{s d}(t)
$$

The problem is solved for each time epoch separately, $t$ for $r_{i}(t)$ and $\gamma_{i j}^{\text {sd }}(t)$ 
M.A.Sc. Thesis - Sayed-Ali Shariatmadari McMaster - Electrical Engineering

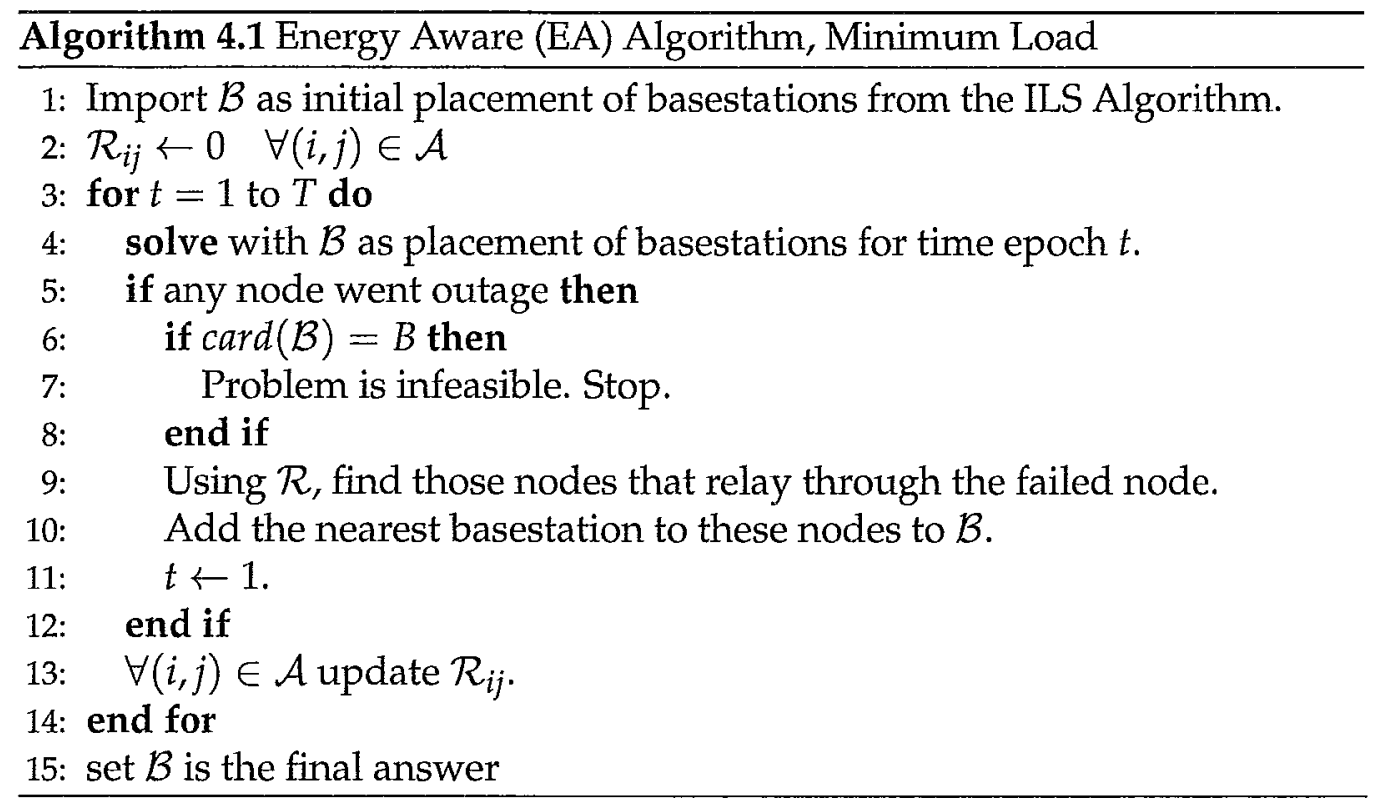

accepts only a single value. To accommodate the above procedure, the optimization objective must be changed. Since activated basestations are known in advance at each time epoch, $\sum_{i \in \mathcal{B}} \lambda_{i}$ is replaced by a linear equation that is energy aware. These changes make the problem a linear program (LP) that is solved very quickly.

\section{Minimum Load}

By replacing $\sum_{i \in \mathcal{B}} \lambda_{i}$ in Equation (4.13) by $\sum_{i \in \mathcal{N}} L_{i}(t)$ the problem becomes a minimum load problem and for a known $t$ the objective becomes,

$$
\text { minimize } \quad w_{1} \sum_{i \in \mathcal{N}} L_{i}(t)+w_{2} \sum_{i \in \mathcal{N}} r_{i}(t)+w_{3} \sum_{\substack{s, d \in \mathcal{F} \\(i, j) \in \mathcal{A}}}^{s d}(t)
$$


M.A.Sc. Thesis - Sayed-Ali Shariatmadari McMaster - Electrical Engineering

This objective acts like a shortest path problem but finds a path with minimum consumption of energy used for transmission. This behavior prevents using multipath routing of data and all the data of a flow travels through the same path. This behavior affects the quality of the solution found by this algorithm as will be shown in Chapter 5 .

\section{Minimum Battery Usage}

Battery usage is defined as the sum of remaining capacity of batteries of all nodes at the current time epoch. By minimizing the battery usage slack variable , $r_{i}(t)$, and the data rate variable, $\gamma_{i j}^{s d}(t)$, are also minimized. Thus there is more need in minimizing them and problem will have only one objective to minimize. In contrast with the minimum Load approach, minimizing of battery usage lets a flow to take different path through the destination and therefore is one of the advantages of this algorithm.

\subsubsection{Initial Placement of Basestations}

The proposed algorithm must start with an initial solution. In order to ensure that we can accommodate large problems, we obtain this using an iterated local search procedure as shown in Algorithm 4.2. The algorithm starts by solving the actual problem when all of the basestations are placed, which is a linear program, and then deactivates unnecessary basestations one by one. For each basestation we are only interested in that particular basestation and therefore the status (placed or not) of all others is not changed. 
M.A.Sc. Thesis - Sayed-Ali Shariatmadari McMaster - Electrical Engineering

The quality of the result depends on the order that basestations are processed and that is why in Line 3 basestations are first sorted based on the number of links that each one has to all of the sensor nodes. This changes the type of the optimization from MILP into a LP feasibility problem, which is even solved much faster than a standard LP problem. Our results for large networks show that this algorithm gives good results and in the cases where we have been able to compute the bound exactly, the algorithm generally performs very well.

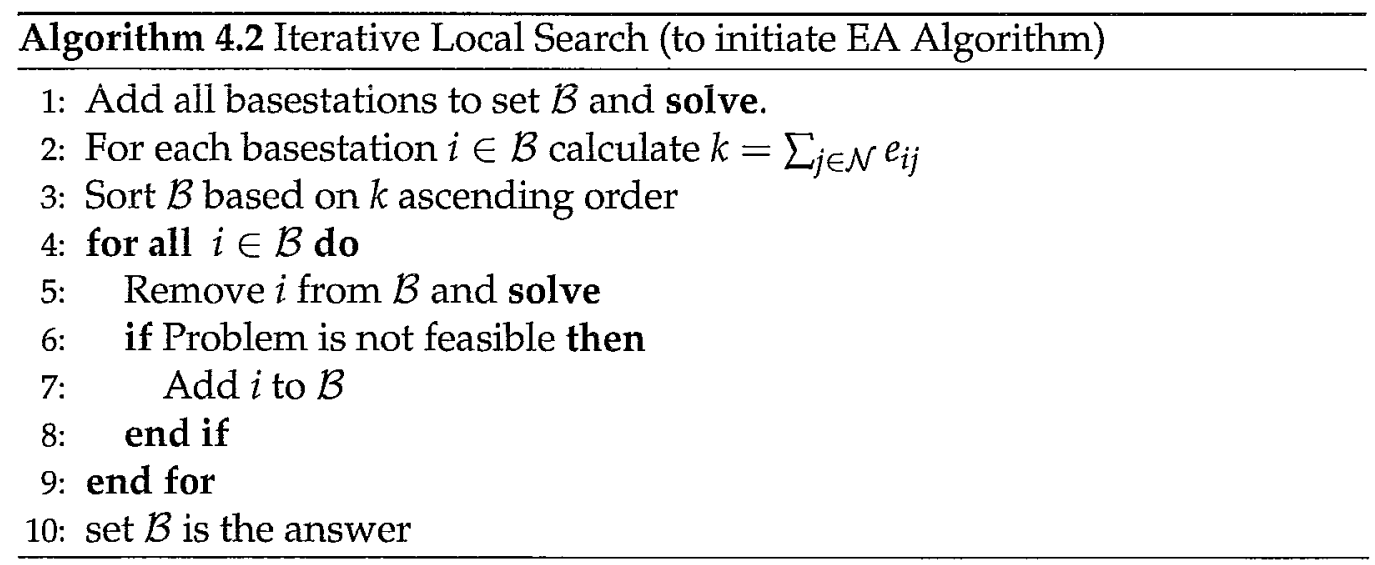

\subsubsection{Complexity of EA Algorithms}

Our algorithm consists of two parts, first a search for an initial placement using Algorithm 4.2 and then Algorithm 4.1 solves the problem using that initial placement, in a causal way. Algorithm 4.1 solves a LP for each time epoch, which is solvable in polynomial time or in other words is of order $O\left(n^{k}\right)$ for some constant $k$. But every time that a node fails with a preselected set of basestations, the loop starts from the beginning. The worst case scenario, only 
M.A.Sc. Thesis - Sayed-Ali Shariatmadari McMaster - Electrical Engineering

one basestation is placed at the start of the algorithm and then every time at the latest time epoch a node fails until all of the basestations are placed. Therefore running time of Algorithm 4.1, for constant $B, T$ and $k$, is upper bounded by $B \times T \times O\left(n^{k}\right)$, which is polynomial. Algorithm 4.2 also solves a LP in each iteration and the total number of iterations is exactly equal to the number of placement for basestations which is constant. This means running time of Algorithm 4.2 is upper bounded by $B \times O\left(n^{k}\right)$ for constant $B$ and $k$, which is also polynomial. This concludes that the total solve time of both EA algorithms is polynomial. 


\section{Chapter 5}

\section{Performance Results}

\subsection{Introduction}

In this chapter we present some representative sample results using the proposed algorithms. Our experiments use solar irradiation data over a 50 day time period based on historical measurements taken in Toronto, Canada. This data was obtained from the Meteorological Service of Canada.

\subsection{Design Example}

We consider a network with 12 sensor nodes using a topology that is shown in Figure 5.1a. Links between nodes and basestations are also shown in Figure 5.1b. Any flow that originates from each node has a normalized value of 0.5 and destined for a basestation. Basestations send a flow with volume of 0.05 unit of traffic to all of the sensor nodes. All of the initial parameters are listed in Table 5.1. 
M.A.Sc. Thesis - Sayed-Ali Shariatmadari McMaster - Electrical Engineering
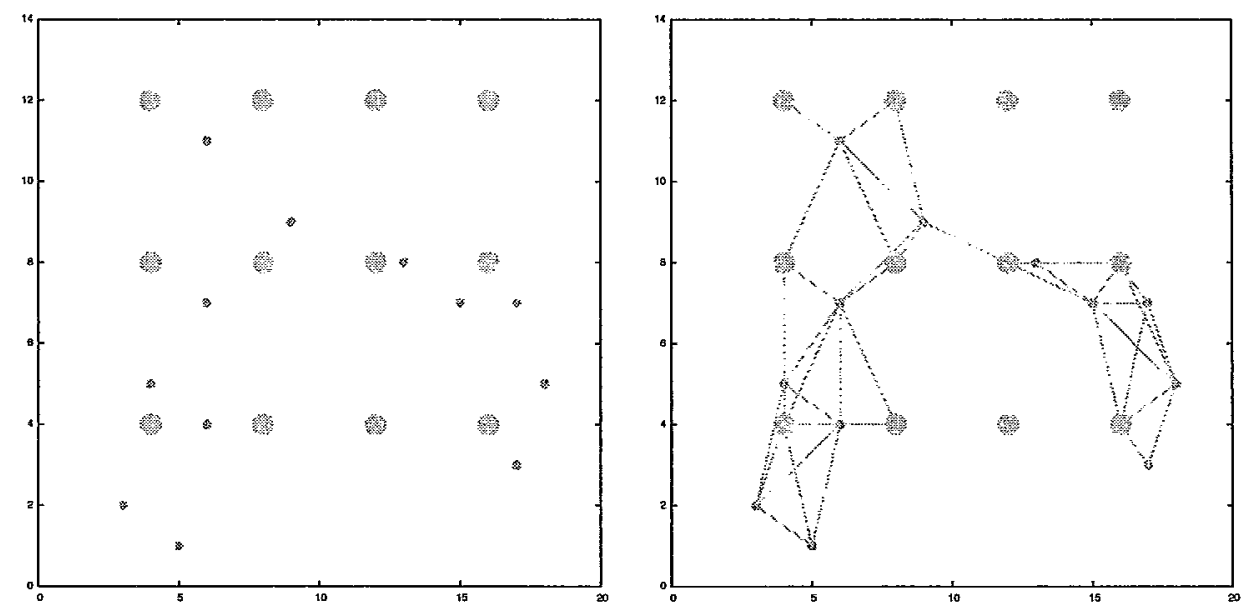

(a) Nodes (circles) and Basestation Candi- (b) Links Between Nodes and Basestations date Locations (squares)

Figure 5.1: Design Example.

The first step for solving this problem is assigning values to $w_{1}, w_{2}$ and $w_{3}$. With the initial parameters that are mentioned in Table 5.1, the number of placed basestations is 3 out of the 12 possible positions. The number of placed basestations changes with parameters such as the solar attenuation factor, link capacity and battery capacity, which are discussed in the following sections.

\subsubsection{The Size of Battery and Solar Panel}

Since sensor nodes that are deployed in a given area usually use the same hardware, our design uses a homogenous battery size and panel size among all nodes. Table 5.2 shows the effect of increasing battery size and panel size on number of basestation. In each case, with a predetermined battery and solar panel, the volume of flows in the network is increased until no optimal solution could be found and then the problem is solved again with having 
M.A.Sc. Thesis - Sayed-Ali Shariatmadari McMaster - Electrical Engineering

\begin{tabular}{r|l}
\hline Candidates for Base Stations & 12 \\
Network Lifetime & 1200 hour \\
Transmission Energy Limit & 0.15 \\
$p_{t, i j}$ & $0.01+0.01 d_{i j}^{2}$ \\
$p_{r, i}$ & 0.01 \\
Panel Size & 4 \\
$B_{\text {outage }}$ & 0.1 \\
$B_{\max (i)}$ & 10 \\
$C_{i j}$ & 1
\end{tabular}

Table 5.1: Modeling Parameters

\begin{tabular}{|c||l|l|l|l|l|l|l|l|l|l|l|l|}
\hline Flow Volume & 0.5 & 0.6 & 0.7 & 0.8 & 0.9 & 1 & 1.1 & 1.2 & 1.3 & 1.4 & 1.5 & 1.6 \\
\hline Battery=2, Panel=1 & 4 & 4 & 5 & - & - & - & - & - & - & - & - & - \\
\hline Battery=5, Panel=1 & 3 & 3 & 4 & 4 & 4 & 5 & 6 & - & - & - & - & - \\
\hline Battery $=10$, Panel $=1$ & 2 & 3 & 3 & 3 & 4 & 4 & 4 & 5 & 5 & 6 & 6 & 8 \\
\hline
\end{tabular}

Table 5.2: The Number of Placed Basestation vs The Volume of Traffic that is Generated by Nodes.

the size of battery or solar panel increased. Table 5.2 clearly shows that the number of basestations are increased when more flow is transmitted through the network. For example, consider the case that $B=2$ and $P=1$ and it can be seen that maximum flow that network can handle is 0.7 unit and 5 basestations out of 12 possible positions are placed. By increasing battery size, $B=5$, maximum flow the network can handle is increased to 1.1 and 6 basestations are placed.

In another example, Table 5.3 shows that in some cases only increasing the size of the battery is not enough and panel size must also be considered in designing the network. By considering $P=1$ and comparing two cases when $B=10$ and $B=20$, one can understand that increasing the size of the battery has a negligible effect on the amount of flow the network can handle and the 
M.A.Sc. Thesis - Sayed-Ali Shariatmadari McMaster - Electrical Engineering

\begin{tabular}{|c||c|l|l|l|l|l|l|l|l|l|l|}
\hline Flow Volume & 1 & 1.1 & 1.2 & 1.3 & 1.4 & 1.5 & 1.6 & 1.7 & 1.8 & 1.9 & 2 \\
\hline Battery=10, Panel=1 & 4 & 4 & 5 & 5 & 6 & 6 & 8 & - & - & - & - \\
\hline Battery=20, Panel=1 & 4 & 4 & 5 & 5 & 5 & 6 & 7 & 8 & - & - & - \\
\hline Battery=20, Panel=3 & 4 & 4 & 4 & 5 & 5 & 5 & 6 & 6 & 7 & 7 & 7 \\
\hline
\end{tabular}

Table 5.3: The Number of Placed Basestation vs The Volume of Traffic that is Generated by Nodes.

number of placed basestations. But when the size of the panel is increased and $P=3$ not only flow with the volume of 2 unit instead of 1.7 unit can be handled but also the number of basestations needed is decreased from 8 to 7 .

\subsubsection{Heterogeneous Solar Factor}

Figure 5.2a shows a network that all of the sensor nodes are harvesting the same amount of solar energy. In other words, the solar factor, $\alpha_{i}(t)$, is homogeneous and has a same value for all nodes $i \in \mathcal{N}$. Figure 5.2a also shows that 3 basestations are enough that the network works for the entire time. In practice, all of the sensor nodes do not harvest the same amount of solar energy at the same time and this affects the number of placed basestations. Figure $5.2 \mathrm{~b}$ shows the same network as in Figure 5.2a but the shaded part receives less solar energy. This change increases the number of placed basestations from 3 to 4 and concentrated more basestations in the shaded area.

\subsubsection{Performance of EA Algorithms}

The results show that decreasing link capacity will increase the number of basestations, which would clearly be expected. For example, by looking at Table 5.4a which shows the Lower Bound, when the solar attenuation factor 
M.A.Sc. Thesis - Sayed-Ali Shariatmadari McMaster - Electrical Engineering
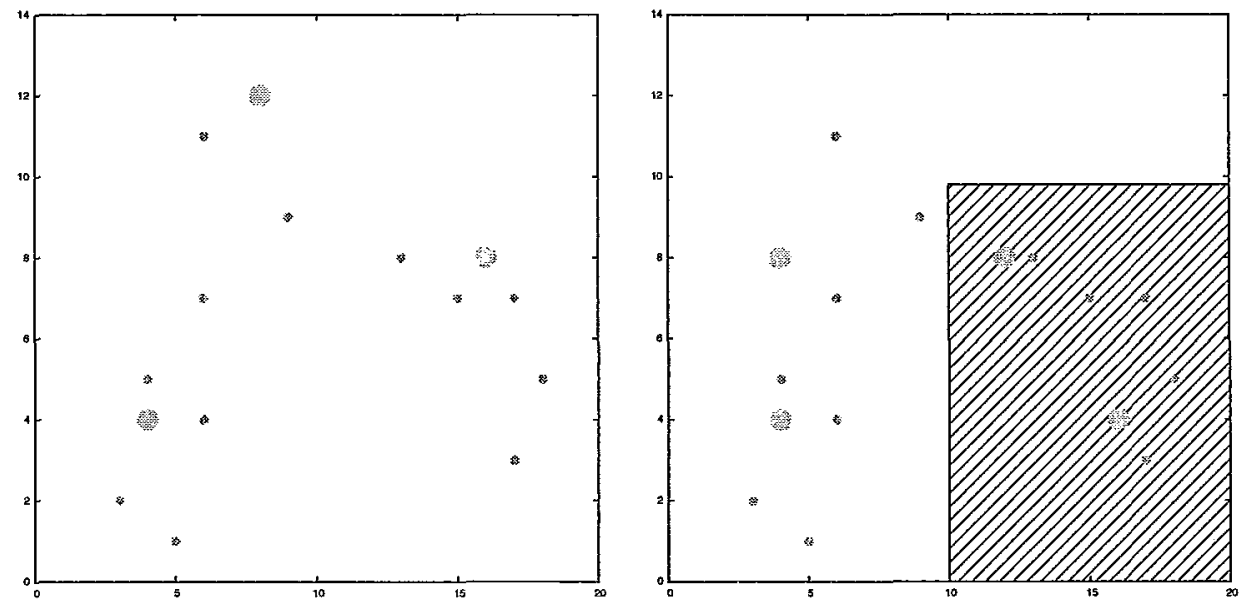

(a) A Network with Homogeneous Solar(b) A Network with Heterogeneous Solar Factor

Factor

Figure 5.2: Comparing Node Placement in a Homogeneous Solar Energy network vs a Heterogeneous one

is 1 , decreasing the link capacity from 1 to 0.3 increases the number of placed basestations from 3 to 6 . This also happens when the battery capacity of the nodes is decreased. Table 5.6a shows that decreasing the battery capacity from 15 to 3 , when the solar attenuation factor is 1 , increases the number of basestations from 3 to 4 . Table 5.4 shows the variation of required basestations when the link capacity and solar attenuation factors change, and Table 5.6 shows the results when the battery capacity and solar attenuation factors are changed. These results were obtained by solving the optimization problem in Equation (4.12) and therefore are lower bounds.

Results are also shown for solutions obtained using the proposed algorithms. Table 5.5a and Table 5.7a show the results obtained using the Minimum Load algorithm and Table $5.5 \mathrm{~b}$ and Table $5.7 \mathrm{~b}$ show results obtained using the Minimum Battery Usage algorithm. Table 5.5a shows that when the 
M.A.Sc. Thesis - Sayed-Ali Shariatmadari McMaster - Electrical Engineering

\begin{tabular}{|c|c|c|c|c|c|c|c|c|}
\cline { 2 - 10 } \multicolumn{1}{c|}{} & \multicolumn{10}{c|}{ Link Capacity } \\
\hline Solar Factor & 0.3 & 0.4 & 0.5 & 0.6 & 0.7 & 0.8 & 0.9 & 1.0 \\
\hline \hline 0.4 & 7 & 5 & 4 & 4 & 4 & 4 & 4 & 4 \\
\hline 0.5 & 7 & 5 & 4 & 4 & 4 & 4 & 4 & 4 \\
\hline 0.6 & 7 & 5 & 4 & 4 & 4 & 4 & 4 & 4 \\
\hline 0.7 & 7 & 5 & 4 & 4 & 4 & 4 & 4 & 4 \\
\hline 0.8 & 7 & 5 & 4 & 4 & 4 & 3 & 3 & 3 \\
\hline 0.9 & 6 & 5 & 4 & 4 & 3 & 3 & 3 & 3 \\
\hline 1.0 & 6 & 5 & 4 & 4 & 3 & 3 & 3 & 3 \\
\hline
\end{tabular}

(a) Bound

\begin{tabular}{|c|c|c|c|c|c|c|c|c|}
\cline { 2 - 10 } \multicolumn{1}{c|}{} & \multicolumn{10}{c|}{ Line Capacity } \\
\hline Solar Factor & 0.3 & 0.4 & 0.5 & 0.6 & 0.7 & 0.8 & 0.9 & 1.0 \\
\hline \hline 0.4 & 7 & 5 & 4 & 4 & 4 & 4 & 4 & 4 \\
\hline 0.5 & 7 & 5 & 4 & 4 & 4 & 4 & 4 & 4 \\
\hline 0.6 & 7 & 5 & 4 & 4 & 4 & 4 & 4 & 4 \\
\hline 0.7 & 7 & 5 & 4 & 4 & 4 & 4 & 4 & 4 \\
\hline 0.8 & 7 & 5 & 4 & 4 & 4 & 3 & 3 & 3 \\
\hline 0.9 & 7 & 5 & 4 & 4 & 3 & 3 & 3 & 3 \\
\hline 1.0 & 7 & 5 & 4 & 4 & 3 & 3 & 3 & 3 \\
\hline
\end{tabular}

(b) ILS Algorithm (input to EA)

Table 5.4: Number of Basestations: Link Capacity vs. Solar Factor. Results of Lower Bound and Local Search Algorithms.

link capacity is 0.4 or 0.5 , the Minimum Load algorithm cannot find a solution. This also happens with low link capacities (i.e., 0.3 ) or low battery capacities (i.e., 3 or 5 ). This happens because we have set a very stringent maximum number of basestations and there may very well be no causal solution to the problem in some cases. Table $5.5 \mathrm{~b}$ and Table $5.7 \mathrm{~b}$ also show that the quality of results obtained using the Minimum Battery Usage algorithm is better. For example, in Table 5.5b, when the link capacity is 0.3, the Minimum Battery Usage algorithm is able find more answers than the Minimum Load algorithm and on average number of placed basestations are lower. 
M.A.Sc. Thesis - Sayed-Ali Shariatmadari McMaster - Electrical Engineering

\begin{tabular}{|c|c|c|c|c|c|c|c|c|}
\cline { 2 - 10 } \multicolumn{1}{c|}{} & \multicolumn{10}{c|}{ Link Capacity } \\
\hline Solar Factor & 0.3 & 0.4 & 0.5 & 0.6 & 0.7 & 0.8 & 0.9 & 1.0 \\
\hline \hline 0.4 & - & - & - & - & - & - & - & - \\
\hline 0.5 & - & - & - & - & - & - & - & - \\
\hline 0.6 & - & 6 & 5 & 5 & 5 & 5 & 5 & 5 \\
\hline 0.7 & - & 6 & 4 & 4 & 4 & 4 & 4 & 4 \\
\hline 0.8 & - & 5 & 4 & 4 & 4 & 4 & 4 & 4 \\
\hline 0.9 & 7 & 5 & 4 & 4 & 4 & 4 & 4 & 4 \\
\hline 1.0 & 7 & 5 & 4 & 4 & 4 & 4 & 4 & 4 \\
\hline
\end{tabular}

(a) EA Algorithm, Minimum Load

\begin{tabular}{|c|c|c|c|c|c|c|c|c|}
\cline { 2 - 10 } \multicolumn{1}{c|}{} & \multicolumn{10}{c|}{ Link Capacity } \\
\hline Solar Factor & 0.3 & 0.4 & 0.5 & 0.6 & 0.7 & 0.8 & 0.9 & 1.0 \\
\hline \hline 0.4 & - & - & - & - & - & - & - & - \\
\hline 0.5 & - & - & - & - & - & - & 8 & 8 \\
\hline 0.6 & 7 & 6 & 5 & 5 & 5 & 5 & 5 & 5 \\
\hline 0.7 & 7 & 5 & 4 & 4 & 4 & 4 & 4 & 4 \\
\hline 0.8 & 7 & 5 & 4 & 4 & 4 & 4 & 4 & 4 \\
\hline 0.9 & 7 & 5 & 4 & 4 & 4 & 4 & 3 & 3 \\
\hline 1.0 & 7 & 5 & 4 & 4 & 4 & 4 & 3 & 3 \\
\hline
\end{tabular}

(b) EA Algorithm, Minimum Battery Usage

Table 5.5: Number of Basestations: Link Capacity vs. Solar Factor. Results of Minimum Load and Minimum Battery Usage Algorithms.

For the same reason, in some of the cases, the output of the EA algorithms slightly exceeds the computed bound. For example, in Table 5.5a, when both link capacity and solar attenuation factors are 1 , the algorithm places 4 basestations while the optimal solution is 3 . However, in general for this and other experiments that have been run, the performance of the EA algorithms tends to be very close to the bound, which is very encouraging. This is achieved by using the Local Serach Algorithm input (Algorithm 4.2) which is very close to the lower bound and yet is computationally tractable. 
M.A.Sc. Thesis - Sayed-Ali Shariatmadari McMaster - Electrical Engineering

\begin{tabular}{|c||c|c|c|c|c|c|c|}
\cline { 2 - 7 } \multicolumn{1}{c|}{} & \multicolumn{7}{c|}{ Battery Capacity } \\
\hline Solar Factor & 3 & 5 & 7 & 9 & 11 & 13 & 15 \\
\hline \hline 0.4 & - & 4 & 4 & 4 & 4 & 4 & 4 \\
\hline 0.5 & 5 & 4 & 4 & 4 & 4 & 3 & 3 \\
\hline 0.6 & 4 & 4 & 4 & 4 & 3 & 3 & 3 \\
\hline 0.7 & 4 & 4 & 4 & 4 & 3 & 3 & 3 \\
\hline 0.8 & 4 & 4 & 4 & 3 & 3 & 3 & 3 \\
\hline 0.9 & 4 & 4 & 4 & 3 & 3 & 3 & 3 \\
\hline 1 & 4 & 4 & 4 & 3 & 3 & 3 & 3 \\
\hline
\end{tabular}

(a) Bound

\begin{tabular}{|c||c|c|c|c|c|c|c|}
\cline { 2 - 8 } \multicolumn{1}{c|}{} & \multicolumn{7}{c|}{ Battery Capacity } \\
\hline Solar Factor & 3 & 5 & 7 & 9 & 11 & 13 & 15 \\
\hline \hline 0.4 & - & 5 & 4 & 4 & 4 & 4 & 4 \\
\hline 0.5 & 5 & 4 & 4 & 4 & 4 & 3 & 3 \\
\hline 0.6 & 5 & 4 & 4 & 4 & 4 & 3 & 3 \\
\hline 0.7 & 4 & 4 & 4 & 4 & 3 & 3 & 3 \\
\hline 0.8 & 4 & 4 & 4 & 3 & 3 & 3 & 3 \\
\hline 0.9 & 4 & 4 & 4 & 3 & 3 & 3 & 3 \\
\hline 1 & 4 & 4 & 4 & 3 & 3 & 3 & 3 \\
\hline
\end{tabular}

(b) ILS Algorithm (input to EA)

Table 5.6: Number of Basestations: Battery Capacity vs. Solar Factor. Results of Lower Bound and Local Search Algorithms.

\subsection{Larger Networks}

In this section the performance of proposed EA algorithms is studied by changing the number of nodes, the amount of traffic they generate and their geographical distribution. Here two distributions are used for positioning the nodes, a uniform distribution and normal distribution, and each distribution is set to generate two volumes of traffic, 1 unit and 0.5 unit, which makes 4 possible networks. In each design the number of nodes is increased from 10 
M.A.Sc. Thesis - Sayed-Ali Shariatmadari McMaster - Electrical Engineering

\begin{tabular}{|c||c|c|c|c|c|c|c|}
\cline { 2 - 8 } \multicolumn{1}{c|}{} & \multicolumn{7}{c|}{ Battery Capacity } \\
\hline Solar Factor & 3 & 5 & 7 & 9 & 11 & 13 & 15 \\
\hline \hline 0.4 & - & - & - & - & - & - & - \\
\hline 0.5 & - & - & - & - & 5 & 5 & 5 \\
\hline 0.6 & - & - & 5 & 5 & 5 & 5 & 5 \\
\hline 0.7 & - & - & 5 & 4 & 4 & 4 & 4 \\
\hline 0.8 & - & 5 & 4 & 4 & 4 & 4 & 4 \\
\hline 0.9 & - & 5 & 4 & 4 & 4 & 4 & 4 \\
\hline 1 & - & 5 & 4 & 4 & 4 & 4 & 4 \\
\hline
\end{tabular}

(a) EA Algorithm, Minimum Load

\begin{tabular}{|c||c|c|c|c|c|c|c|}
\cline { 2 - 8 } \multicolumn{1}{c|}{} & \multicolumn{6}{c|}{ Battery Capacity } \\
\hline Solar Factor & 3 & 5 & 7 & 9 & 11 & 13 & 15 \\
\hline \hline 0.4 & - & - & - & - & - & 8 & 7 \\
\hline 0.5 & - & - & - & 7 & 5 & 5 & 5 \\
\hline 0.6 & - & 5 & 5 & 5 & 5 & 5 & 5 \\
\hline 0.7 & - & 5 & 5 & 4 & 4 & 4 & 4 \\
\hline 0.8 & - & 5 & 4 & 4 & 4 & 3 & 3 \\
\hline 0.9 & 7 & 5 & 4 & 4 & 3 & 3 & 3 \\
\hline 1 & 7 & 5 & 4 & 4 & 3 & 3 & 3 \\
\hline
\end{tabular}

(b) EA Algorithm, Minimum Battery Usage

Table 5.7: Number of Basestations: Battery Capacity vs. Solar Factor. Results of Minimum Load and Minimum Battery Usage Algorithms.

to 70 . Each data point that is plotted, is the average of 10 different node positions, for example, when the flow is supposed to be 1 unit, the number of nodes are 70 and the distribution of nodes are uniform, 10 different uniformly distributed positioned are selected, solved and then their results are averaged.

\subsubsection{Geographical Distribution of Sensor Nodes}

Figure 5.3 shows two distributions that are studied, Uniform distribution and Normal distribution. Nodes with the normal distribution are first generated 
M.A.Sc. Thesis - Sayed-Ali Shariatmadari McMaster - Electrical Engineering

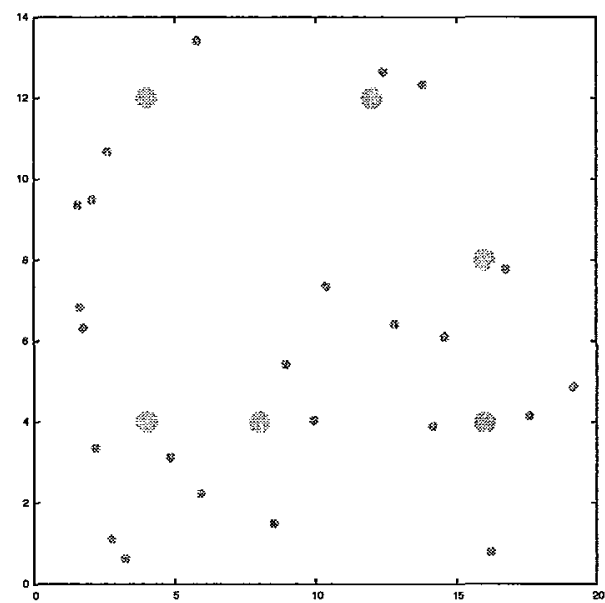

(a) Uniform Distribution

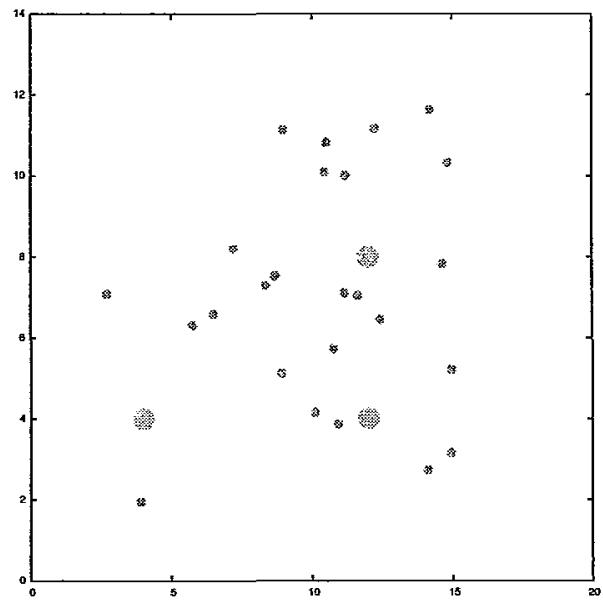

(b) Normal Distribution

Figure 5.3: Different Network Topologies

using $\rho=0.5$ and $\sigma=0.2$ and then scaled to the size of the field. Figure 5.3 shows that when the distribution of nodes is normal fewer numbers of basestations are placed, on the other hand, on average more time is spend solving the problem. This happens because in a normally distributed network, nodes are near each other and the cost of communication and the relaying of data is much less than a uniformly distributed network, which has a higher average distance between nodes. Relying more on data relaying also makes solving the problem for the normally distributed network harder, therefore it takes more time for the solver to find the optimal solution.

\subsubsection{Solving Time}

As mentioned before, the problem is a Mixed Integer Linear Programming problem, which is NP-Hard. Figure 5.4 makes comparing the solving time of 
M.A.Sc. Thesis - Sayed-Ali Shariatmadari McMaster - Electrical Engineering

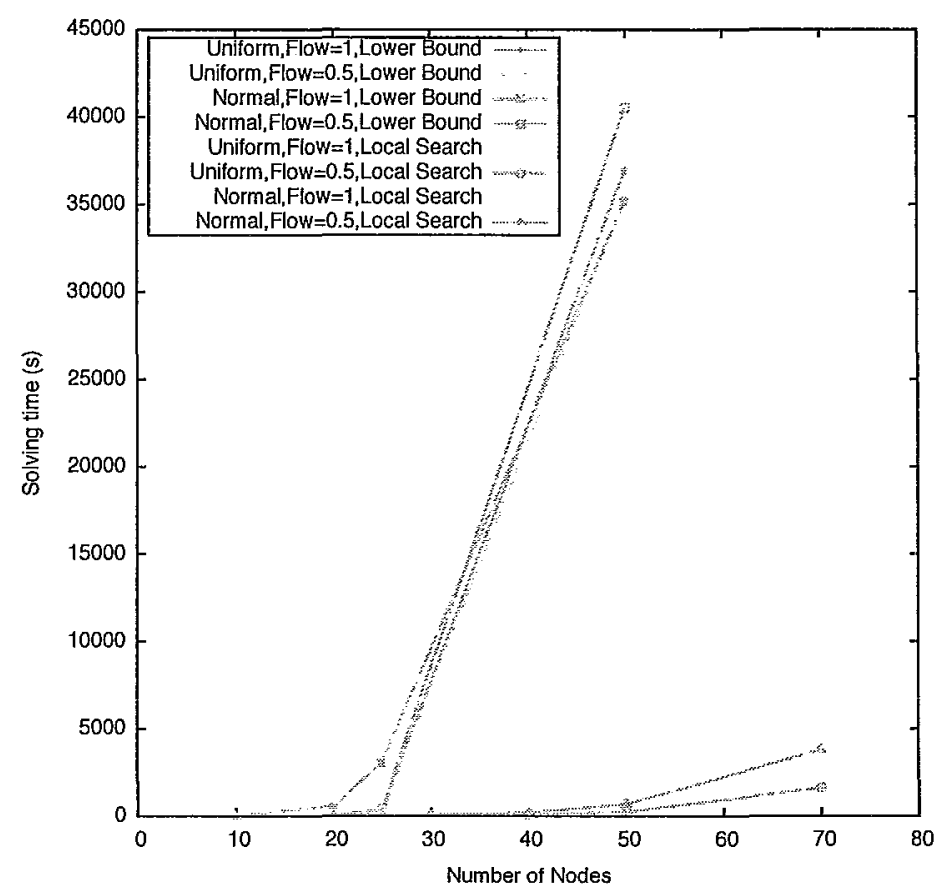

Figure 5.4: Problem Solve Time of Different Topologies and Flow Volume

Lower Bound and Local Search algorithms easy. It can be seen that the solve time of the Lower bound problem is increased exponentially compared to the solve time of the Local Search algorithm that is very reasonable. Adding this to the near optimal results of the Local Search algorithm makes this algorithm a very good replacement for the Lower Bound algorithm.

Another advantage of the Local Search algorithm is that the solve time of different network positions with same number of nodes is about the same. Table 5.8 shows the coefficient of variation of solve time for different numbers of nodes and topologies. It shows that the solving time of the Lower Bound algorithm varies a lot, sometimes even two times the average. On the other hand, the solving time of the Local Search algorithm varies much less and this 
M.A.Sc. Thesis - Sayed-Ali Shariatmadari McMaster - Electrical Engineering

\begin{tabular}{|r|l|l|l|l|l|l|l|}
\hline Number of nodes & 10 & 20 & 25 & 30 & 40 & 50 & 70 \\
\hline Uniform,Lower Bound & 0.62 & 1.11 & 1.25 & - & - & 0.89 & - \\
\hline Normal,Lower Bound & 0.63 & 0.90 & 0.84 & - & - & 0.63 & - \\
\hline Uniform,Local Search & 0.17 & 0.08 & 0.09 & 0.14 & 0.20 & 0.32 & 0.36 \\
\hline Normal,Local Search & 0.15 & 0.10 & 0.08 & 0.18 & 0.25 & 0.17 & 0.15 \\
\hline
\end{tabular}

Table 5.8: The Coefficient of Variation of Solving Time

is persistent for different number of nodes and different distribution of nodes positions.

\subsubsection{Results}

Figure 5.5 shows the average number of placed basestations using the Lower Bound, Local Search, Minimum Load and Minimum Battery Usage algorithms when nodes are distributed uniformly and flow volume is 1 unit of traffic. As mentioned earlier, each data point is the average of 10 problems with different node positions. This setup needed the highest number of basestations on average compared to the other network setups, because first of all, a network, where nodes are distributed uniformly, needs more basestations on average than a network with Normal node distribution and secondly, a uniformly distributed network that is supposed to handle 1 unit of traffic is the most difficult case for EA algorithms, studied here. Figure 5.5 also approves the quality of results that the Local Search algorithm generates. It also can be noticed that compared to the Lower Bound algorithm, both EA algorithms place a higher number of basestations in this setup, with a slight advantage to the Minimum Battery Usage algorithm.

The main advantage of the Minimum Battery Usage algorithm here can be 


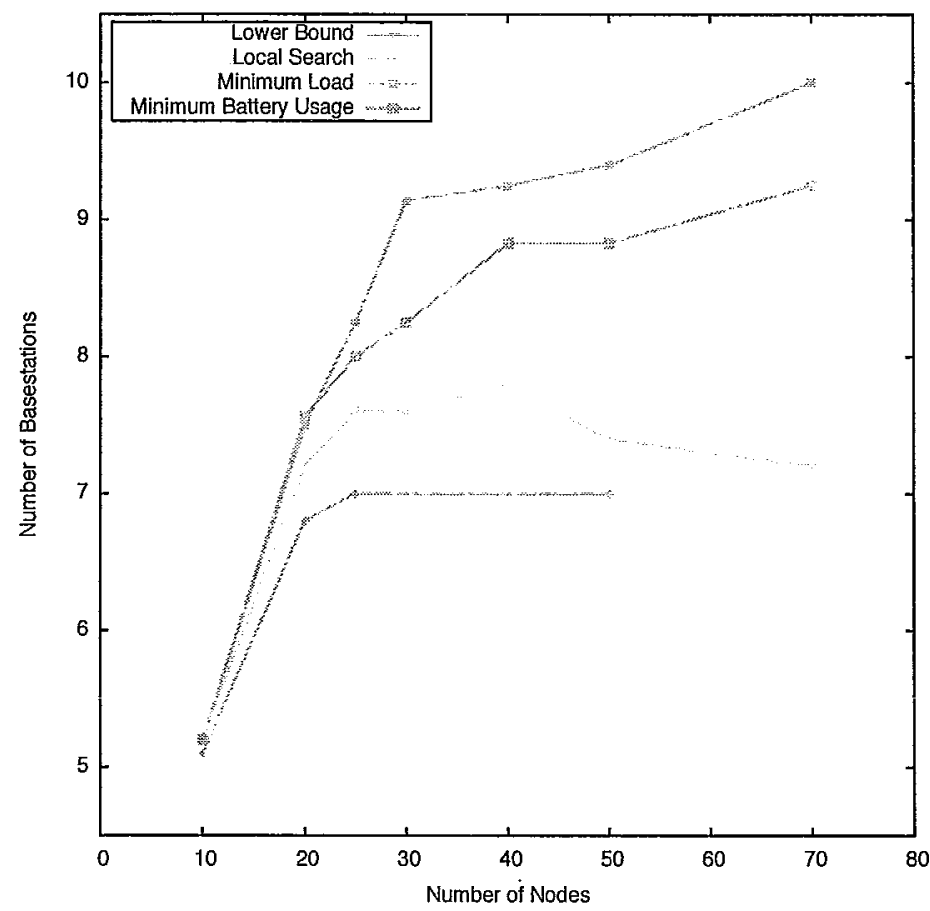

Figure 5.5: Results for a Uniform Distribution when Flow Volume is 1 unit

\begin{tabular}{|r|l|l|l|l|l|l|l|}
\hline Number of nodes & 10 & 20 & 25 & 30 & 40 & 50 & 70 \\
\hline Minimum Load & $100 \%$ & $80 \%$ & $80 \%$ & $70 \%$ & $40 \%$ & $50 \%$ & $30 \%$ \\
\hline Minimum Battery Usage & $100 \%$ & $90 \%$ & $80 \%$ & $80 \%$ & $60 \%$ & $60 \%$ & $40 \%$ \\
\hline
\end{tabular}

Table 5.9: Degree of competence of two EA algorithms

seen in Table 5.9, which shows a number of cases that EA algorithms were successful in finding an answer to the problem. On smaller networks both algorithms do a very good job in finding a solution and these solutions, as seen on Figure 5.5, are near optimal. But as can be seen, on larger networks between two EA algorithms, the Minimum Battery Usage algorithm does a better job than the Minimum Load algorithm. On a network with 40 nodes, Minimum Load was able to find solutions for the $40 \%$ of cases modeled, in contrast to 
M.A.Sc. Thesis - Sayed-Ali Shariatmadari McMaster - Electrical Engineering

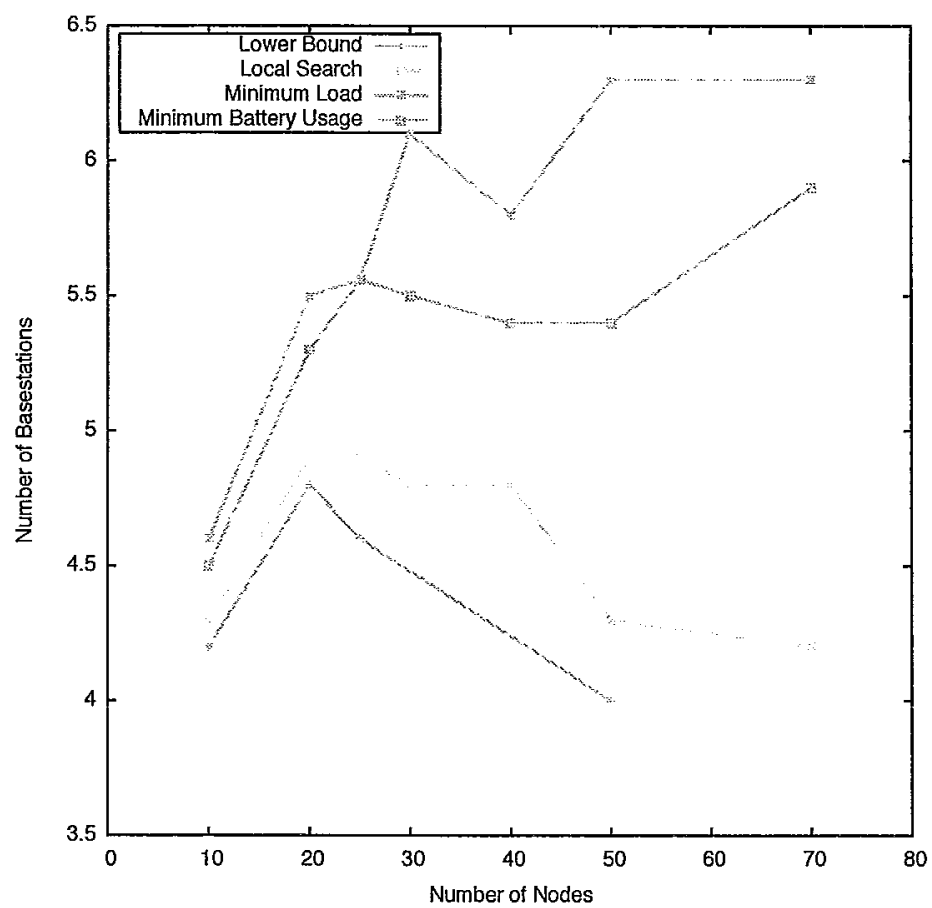

Figure 5.6: Results for a Uniform Distribution when Flow Volume is 0.5 unit

the Minimum Battery Usage algorithm that found solutions for the $60 \%$ of the cases. This comes from the fact that the outcome of Minimum Battery Usage algorithm is better and the average number of basestations placed by the Minimum Battery Usage algorithm is less than the average number of basestations placed by the Minimum Load algorithm.

Figure 5.6 shows the number of placed basestations for a network where nodes are distributed uniformly and the amount of generated flow is 0.5 unit. By comparing these results to the previous case, Figure 5.5, one can understand that fewer numbers of basestations are placed, which makes sense. Figure 5.6 also shows that by increasing the number of nodes in the network beyond a threshold, the number of basestations placed by the Lower Bound 


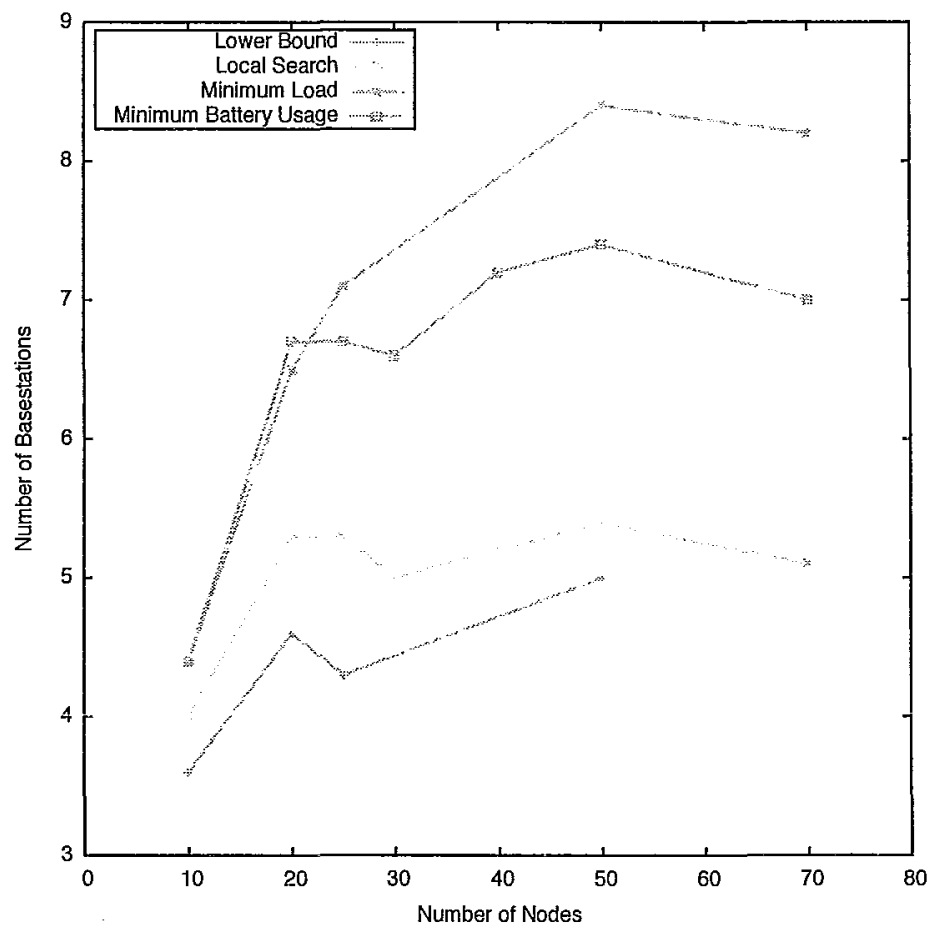

Figure 5.7: Results for a Normal Distribution when Flow Volume is 1 unit

algorithm is decreased. This phenomena happens because with more nodes, there is more opportunity for the existence of a node with enough energy to relay data and this helps the Lower Bound algorithm to use nodes to relay data instead of placing a new basestation. Even though this wasn't happening on a network with 1 unit of traffic, Figure 5.5, because in the network with the higher volume of flows, nodes eventually spend more energy to transmit data and this higher cost of data transmission is more than the amount of energy that each node harvests and stores in its battery. This reduces the oppertunity for each node to realy traffic for other nodes and finally it leads to higher number of basestations.

It also can be seen that the Local Search algorithm follows the lower bound 
M.A.Sc. Thesis - Sayed-Ali Shariatmadari McMaster - Electrical Engineering

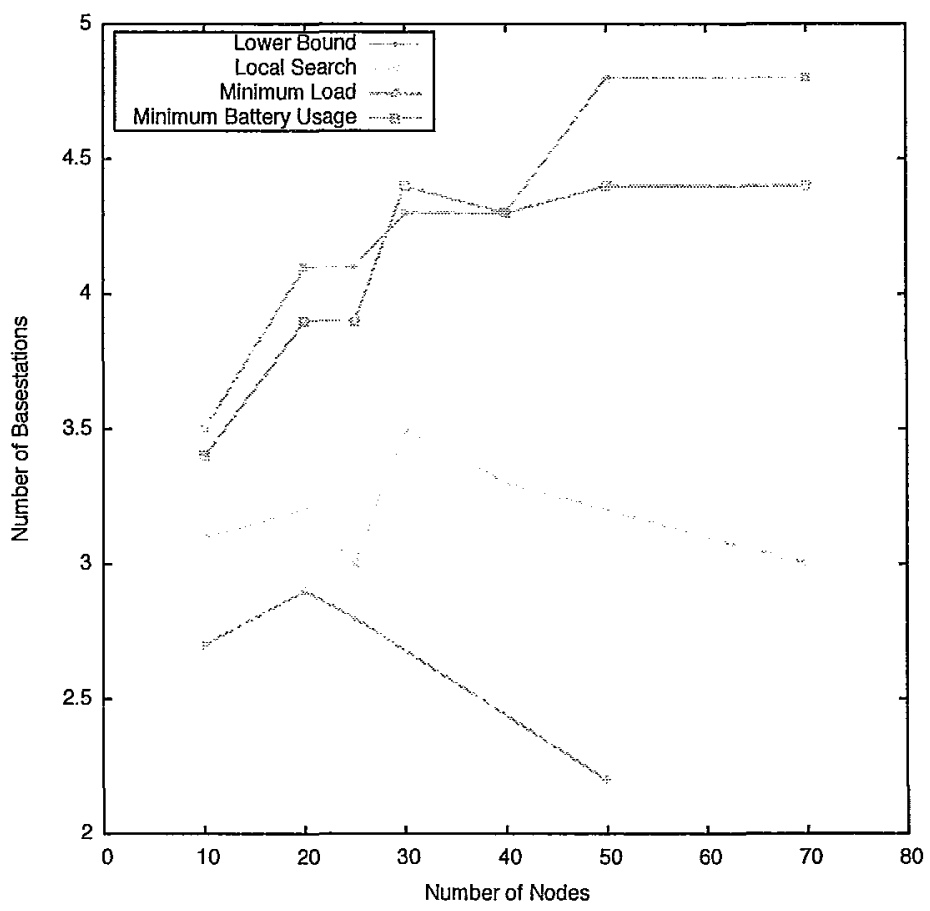

Figure 5.8: Results for a Normal Distribution when Flow Volume is 0.5 unit

and places fewer basestations, which helps EA algorithms to be able to find solutions for all of the case studies. The Minimum Battery Usage algorithm showed better performance in larger networks, even though both by increasing the number of nodes distance between the lower bound and the results of EA algorithms increases. This happens because in large networks, optimal results rely heavily on relaying of data by nodes, instead of placing a new basestation and this makes finding a causal solution without having the data of future energy harvesting very hard.

Figure 5.7 shows placed basestations for networks that nodes are positioned by the Normal distribution and generate 1 unit of traffic. Compare to the uniformly distributed networks, Figure 5.5, normally distributed networks 
M.A.Sc. Thesis - Sayed-Ali Shariatmadari McMaster - Electrical Engineering

need fewer basestations. The proposed algorithms also were able to find solutions for all of the case studies and the quality of results is reasonably good. The better performance of Minimum Battery Usage algorithm is also repeated in this network type either.

Figure 5.8 shows the same networks when flow generated by nodes are 0.5 unit. As can be seen from the figure, on average, both EA algorithms place fewer basestations and the quality of their result are about the same. This is because, on average, a normally distributed network with flow volume of 0.5 unit needs the least number of basestations among the cases studied here.

These results suggest that using only energy aware shortest path routing, i.e. Minimizing Load, is not always the best solution for such a network. Incorporating a smart routing algorithm that has the ability to model and estimate the input energy of the nodes can improve the performance. 


\section{Chapter 6}

\section{Conclusion and Future Works}

In this thesis we have considered the minimum-cost placement of data collecting basestation nodes so that outage-free operation of solar powered sensor nodes is obtained. This was accomplished by minimizing the number of basestations required when taking into account the energy renewal rates and costs of sensor node traffic relaying. An optimization was formulated which gives a lower bound on the number of basestations that are needed. Because of the complexity of the problem, the bound can only be found for fairly small problem sizes. To deal with the complexity issue, an algorithm was proposed which can be used to do placements for practical problems. The algorithm uses the result from an iterated local search algorithm as a starting point, and then uses an energy aware local optimization to obtain feasible basestation placements. Results were presented which show that the algorithm performs well for different network scenarios and parameter values.

For Future work, one can make the basestations battery or solar powered instead of considering them having unlimited power. These basestations will 
M.A.Sc. Thesis - Sayed-Ali Shariatmadari McMaster - Electrical Engineering

need battery and panel size design which defines new problems, such as provisioning. Another idea is to improve the performance of the heuristic algorithms proposed here by exploiting an estimation of future solar energy. This can be done by modeling the environmental energy mathematically and using statistical information to estimate it. 


\section{Bibliography}

[1] S. Roundy, P. Wright, and J. Rabaey, Energy Scavenging for Wireless Sensor Networks: With Special Focus on Vibrations. Springer, 2004.

[2] D. Estrin, R. Govindan, and J. Heidemann, "Embedding the Internet: Introduction," Commun. ACM, vol. 43, no. 5, pp. 38-41, 2000.

[3] M. Srivastava, R. Muntz, and M. Potkonjak, "Smart Kindergarten: SensorBased Wireless Networks for Smart Developmental Problem-Solving Environments," in MobiCom '01: Proceedings of the 7th annual international conference on Mobile computing and networking, 2001, pp. 132-138.

[4] M. Younis, M. Youssef, and K. Arisha, "Energy-Aware Management for Cluster-Based Sensor Networks," Computer Networks, vol. 43, no. 5, pp. 649-668, 2003.

[5] I. F. Akyildiz, W. Su, Y. Sankarasubramaniam, and E. Cayirci, "Wireless Sensor Networks: A Survey," Computer Networks, vol. 38, no. 4, pp. $393-$ $422,2002$.

[6] Y. Shi and Y. Hou, "Approximation Algorithm for Base Station Placement in Wireless Sensor Networks," in 4th Annual IEEE Communications Society 
M.A.Sc. Thesis - Sayed-Ali Shariatmadari McMaster - Electrical Engineering

Conference on Sensor, Mesh and Ad Hoc Communications and Networks, 2007. SECON '07, June 2007, pp. 512-519.

[7] A. A. Sayegh, T. D. Todd, and M. N. Smadi, "Resource Allocation and Cost in Hybrid Solar/Wind Powered WLAN Mesh Nodes," Wireless Mesh Networks: Architectures and Protocols, Springer Science and Business Media Inc., 2008.

[8] A. Farbod and T. Todd, "Resource Allocation and Outage Control for Solar-Powered WLAN Mesh Networks," IEEE Transactions on Mobile Computing, vol. 6, no. 8, pp. 960-970, 2006.

[9] A. Kansal, J. Hsu, S. Zahedi, and M. B. Srivastava, "Power Management in Energy Harvesting Sensor Networks," ACM Trans. Embed. Comput. Syst., vol. 6, no. 4, pp. 32-70, 2007.

[10] S. Boyd and L. Vandenberghe, Convex Optimization. Cambridge Univ Pr, 2004.

[11] Z.-Q. Luo and W. Yu, "An Introduction to Convex Optimization for Communications and Signal Processing," IEEE Journal on Selected Areas in Communications, vol. 24, no. 8, pp. 1426-1438, Aug. 2006.

[12] C. Papadimitriou and K. Steiglitz, Combinatorial Optimization: Algorithms and Complexity. Dover Pubns, 1998.

[13] "IBM ILOG CPLEX Optimizer." [Online]. Available: http://www-01. ibm.com/software/integration/optimization/cplex-optimizer/

[14] "Gurobi Optimizer." [Online]. Available: http://gurobi.com/ 
M.A.Sc. Thesis - Sayed-Ali Shariatmadari McMaster - Electrical Engineering

[15] S. A. Shariatmadari, A. A. Sayegh, and T. D. Todd, "Energy Aware Basestation Placement in Solar Powered Sensor Networks," in IEEE Wireless Communications and Networking Conference (WCNC), 2010, Apr. 2010, pp. 1 $-6$.

[16] J. Hill and D. Culler, "Mica: A Wireless Platform for Deeply Embedded Networks," IEEE Micro, vol. 22, no. 6, pp. 12-24, Nov. 2002.

[17] A. Mainwaring, D. Culler, J. Polastre, R. Szewczyk, and J. Anderson, "Wireless Sensor Networks for Habitat Monitoring," in WSNA '02: Proceedings of the 1st ACM international workshop on Wireless sensor networks and applications, 2002, pp. 88-97.

[18] F. Hu, S. Lakdawala, Q. Hao, and M. Qiu, "Low-Power, Intelligent Sensor Hardware Interface for Medical Data Preprocessing," IEEE Transactions on Information Technology in Biomedicine, vol. 13, no. 4, pp. 656 -663, Jul. 2009.

[19] G. Mathur, P. Desnoyers, P. Chukiu, D. Ganesan, and P. Shenoy, “UltraLow Power Data Storage for Sensor Networks," ACM Trans. Sen. Netw., vol. 5, no. 4, pp. 1-34, 2009.

[20] S. Slijepcevic and M. Potkonjak, "Power Efficient Organization of Wireless Sensor Networks," in IEEE International Conference on Communications, 2001. ICC 2001, vol. 2, 2001, pp. $472-476$.

[21] "Simulating TinyOS Networks." [Online]. Available: http://www.cs. berkeley.edu/ pal/research/tossim.html 
M.A.Sc. Thesis - Sayed-Ali Shariatmadari McMaster - Electrical Engineering

[22] V. Shnayder, M. Hempstead, B.-r. Chen, G. W. Allen, and M. Welsh, "Simulating the Power Consumption of Large-Scale Sensor Network Applications," in SenSys '04: Proceedings of the 2nd international conference on Embedded networked sensor systems, 2004, pp. 188-200.

[23] S. Cui, A. Goldsmith, and A. Bahai, "Energy-Efficiency of Mimo and Cooperative Mimo Techniques in Sensor Networks," IEEE Journal on Selected Areas in Communications, vol. 22, no. 6, pp. 1089 - 1098, Aug. 2004.

[24] G. Zhou, T. He, S. Krishnamurthy, and J. A. Stankovic, "Impact of Radio Irregularity on Wireless Sensor Networks," in MobiSys '04: Proceedings of the 2 nd international conference on Mobile systems, applications, and services, 2004, pp. 125-138.

[25] H. O. Tan and I. Körpeoğlu, "Power Efficient Data Gathering and Aggregation in Wireless Sensor Networks," SIGMOD Rec., vol. 32, no. 4, pp. 66-71, 2003.

[26] B. Hohlt, L. Doherty, and E. Brewer, "Flexible Power Scheduling for Sensor Networks," in IPSN '04: Proceedings of the 3rd international symposium on Information processing in sensor networks, 2004, pp. 205-214.

[27] N. Burri, P. von Rickenbach, and R. Wattenhofer, "Dozer: Ultra-Low Power Data Gathering in Sensor Networks," in IPSN '07: Proceedings of the 6th international conference on Information processing in sensor networks, 2007, pp. 450-459. 
M.A.Sc. Thesis - Sayed-Ali Shariatmadari McMaster - Electrical Engineering

[28] M. Younis, M. Youssef, and K. Arisha, "Energy-Aware Routing in ClusterBased Sensor Networks," in 10th IEEE International Symposium on Modeling, Analysis and Simulation of Computer and Telecommunications Systems, 2002. MASCOTS 2002. Proceedings, 2002, pp. $129-136$.

[29] M. Younis and K. Akkaya, "Strategies and Techniques for Node Placement in Wireless Sensor Networks: A Survey," Ad Hoc Netw., vol. 6, no. 4, pp. 621-655, Jan 2008.

[30] M. Younis, M. Bangad, and K. Akkaya, "Base-Station Repositioning for Optimized Performance of Sensor Networks," in IEEE 58th Vehicular Technology Conference, 2003. VTC 2003-Fall. 2003, vol. 5, Oct. 2003, pp. 2956 2960.

[31] K. Akkaya and M. Younis, "Energy-Aware Routing to a Mobile Gateway in Wireless Sensor Networks," in IEEE Global Telecommunications Conference Workshops, 2004. GlobeCom Workshops 2004, Nov. 2004, pp. 16-21.

[32] Y. Hou, Y. Shi, H. Sherali, and S. Midkiff, "On Energy Provisioning and Relay Node Placement for Wireless Sensor Networks," IEEE Transactions on Wireless Communications, vol. 4, no. 5, pp. 2579-2590, Sep. 2005.

[33] J. Pan, Y. T. Hou, L. Cai, Y. Shi, and S. X. Shen, "Topology Control for Wireless Sensor Networks," in Proceedings of the 9th annual international conference on Mobile computing and networking, ser. MobiCom '03, 2003, pp. 286-299.

[34] A. Bogdanov, E. Maneva, and S. Riesenfeld, "Power-Aware Base Station 
M.A.Sc. Thesis - Sayed-Ali Shariatmadari McMaster - Electrical Engineering

Positioning for Sensor Networks," in Twenty-third AnnualJoint Conference of the IEEE Computer and Communications Societies. INFOCOM 2004, vol. 1, Mar. 2004, pp. $575-585$.

[35] A. Efrat, S. Har-Peled, and J. Mitchell, "Approximation Algorithms for Two Optimal Location Problems in Sensor Networks," in 2nd International Conference on Broadband Networks, 2005. BroadNets 2005, vol. 1, Oct. 2005, pp. $714-723$.

[36] Y. Shi and Y. T. Hou, "Optimal Base Station Placement in Wireless Sensor Networks," ACM Trans. Sen. Netw., vol. 5, no. 4, pp. 1-24, 2009.

[37] A. Farbod, "Design and Resource Allocation for Solar-Powered Ess Mesh Networks," Master's thesis, McMaster University, 2005.

[38] S. Wright, D. Scott, J. Haddow, and M. Rosen, "The Upper Limit to Solar Energy Conversion," in 35th Intersociety Energy Conversion Engineering Conference and Exhibit, 2000. (IECEC), vol. 1, 2000, pp. $384-392$.

[39] V. Raghunathan, A. Kansal, J. Hsu, J. Friedman, and M. Srivastava, "Design Considerations for Solar Energy Harvesting Wireless Embedded Systems," in IPSN '05: Proceedings of the 4th international symposium on Information processing in sensor networks, Apr. 2005, pp. 457 - 462.

[40] X. Jiang, J. Polastre, and D. Culler, "Perpetual Environmentally Powered Sensor Networks," in Fourth International Symposium on Information Processing in Sensor Networks, 2005. IPSN 2005, Apr. 2005, pp. 463 - 468. 
M.A.Sc. Thesis - Sayed-Ali Shariatmadari McMaster - Electrical Engineering

[41] J. Jeong, X. Jiang, and D. Culler, "Design and Analysis of Micro-Solar Power Systems for Wireless Sensor Networks," in 5th International Conference on Networked Sensing Systems, 2008. INSS 2008, Jun. 2008, pp. 181 -188 .

[42] M. Rahimi, H. Shah, G. Sukhatme, J. Heideman, and D. Estrin, "Studying the Feasibility of Energy Harvesting in a Mobile Sensor Network," in IEEE International Conference on Robotics and Automation, 2003. Proceedings. ICRA '03, vol. 1, Sep. 2003, pp. $19-24$.

[43] P. Corke, P. Valencia, P. Sikka, T. Wark, and L. Overs, "Long-Duration Solar-Powered Wireless Sensor Networks,' in EmNets '07: Proceedings of the 4th workshop on Embedded networked sensors, 2007, pp. 33-37.

[44] S. Singh, M. Woo, and C. S. Raghavendra, "Power-Aware Routing in Mobile Ad Hoc Networks," in MobiCom '98: Proceedings of the 4th annual ACM/IEEE international conference on Mobile computing and networking, 1998, pp. 181-190.

[45] A. Sinha and A. Chandrakasan, "Dynamic Power Management in Wireless Sensor Networks," IEEE Design Test of Computers, vol. 18, no. 2, pp. 62-74, Mar. 2001.

[46] A. Kansal and M. Srivastava, "An Environmental Energy Harvesting Framework for Sensor Networks," in Proceedings of the 2003 International Symposium on Low Power Electronics and Design, 2003. ISLPED '03, Aug. 2003, pp. $481-486$. 
M.A.Sc. Thesis - Sayed-Ali Shariatmadari McMaster - Electrical Engineering

[47] T. Voigt, H. Ritter, and J. Schiller, "Solar-Aware Routing in Wireless Sensor Networks," in Personal Wireless Communications, ser. Lecture Notes in Computer Science. Springer Berlin / Heidelberg, 2003, vol. 2775, pp. $847-852$.

[48] C. Bergonzini, D. Brunelli, and L. Benini, "Algorithms for Harvested Energy Prediction in Batteryless Wireless Sensor Networks," in 3rd International Workshop on Advances in sensors and Interfaces, 2009. IWASI 2009, Jun. 2009, pp. $144-149$.

[49] V. Pryyma, L. Bölöni, and D. Turgut, "Uniform Sensing Protocol for Aütonomous Rechargeable Sensor Networks," in MSWiM '08: Proceedings of the 11th international symposium on Modeling, analysis and simulation of wireless and mobile systems, 2008, pp. 92-99.

[50] S. Olariu, M. Eltoweissy, and M. Younis, "ANSWER: Autonomous Wireless Sensor Network," in Q2SWinet '05: Proceedings of the 1st ACM international workshop on Quality of service \& security in wireless and mobile networks, 2005, pp. 88-95.

[51] D. Noh, D. Lee, and H. Shin, "QoS-Aware Geographic Routing for SolarPowered Wireless Sensor Networks," IEICE Transactions, vol. 90-B, no. 12, pp. 3373-3382, 2007.

[52] "The SolarMESH Network," McMaster University. Hamilton, Ontario, Canada, 2004. [Online]. Available: http:/ /owl.mcmaster.ca/solarmesh/

[53] F. Zhang, T. D. Todd, D. Zhao, and V. Kezys, "Power Saving Access Points 
$\underline{\text { M.A.Sc. Thesis - Sayed-Ali Shariatmadari McMaster - Electrical Engineering }}$

for IEEE 802.11 Wireless Network Infrastructure," IEEE Transactions on Mobile Computing, vol. 5, no. 2, pp. 144-156, 2006.

[54] Y. Li, T. Todd, and D. Zhao, "Access Point Power Saving in Solar/Battery Powered IEEE 802.11 ESS Mesh Networks," in Second International Conference on Quality of Service in Heterogeneous Wired/Wireless Networks, 2005, Aug. 2005, pp. $5-49$.

[55] A. Sayegh, S. Ghosh, and T. Todd, "Optimal Node Placement in Hybrid Solar Powered WLAN Mesh Networks," in IEEE Wireless Communications and Networking Conference, 2008. WCNC 2008, Mar. 2008, pp. 2277-2282.

[56] G. Badawy, A. Sayegh, and T. Todd, "Solar Powered Wlan Mesh Network Provisioning for Temporary Deployments," in IEEE Wireless Communications and Networking Conference, 2008. WCNC 2008, Mar. 2008, pp. 2271 -2276 .

[57] —_ "Energy Aware Provisioning in Solar Powered WLAN Mesh Networks," in Proceedings of 17th International Conference on Computer Communications and Networks, 2008. ICCCN '08, Aug. 2008, pp. $1-6$.

[58] A. Mohsenian-Rad, "Optimal Resource Management in Wireless Access Networks," Ph.D. dissertation, The University of British Columbia, 2008.

[59] F. Glover and G. Kochenberger, Handbook of Metaheuristics. Springer, 2003. 